\title{
Analyse secondaire des données de l'analyse situationnelle des services de santé de la reproduction
}

Gisele Kaboré

Population Council

Idrissa Kabore

Follow this and additional works at: https://knowledgecommons.popcouncil.org/departments_sbsr-pgy

Part of the Demography, Population, and Ecology Commons, Family, Life Course, and Society

Commons, Gender and Sexuality Commons, International Public Health Commons, and the Medicine and Health Commons

How does access to this work benefit you? Let us know!

\section{Recommended Citation}

Kaboré, Gisele and Idrissa Kabore. 2009. "Analyse secondaire des données de l'analyse situationnelle des services de santé de la reproduction," Rapport. Ouagadougou: Population Council. 

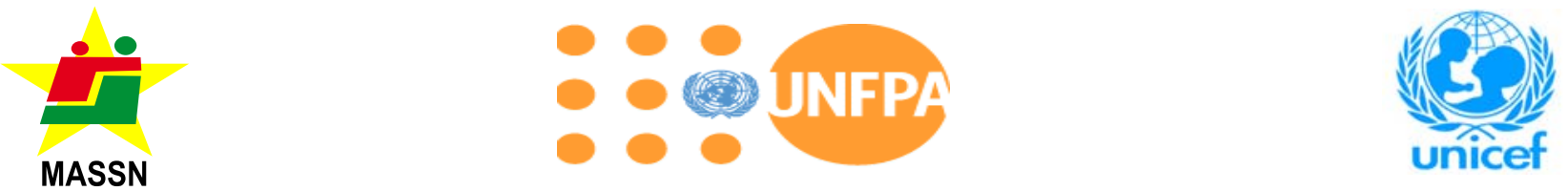

Projet : " Eliminer le mariage des enfants au Burkina : un plan pour la protection, le renforcement et l'action communautaire »
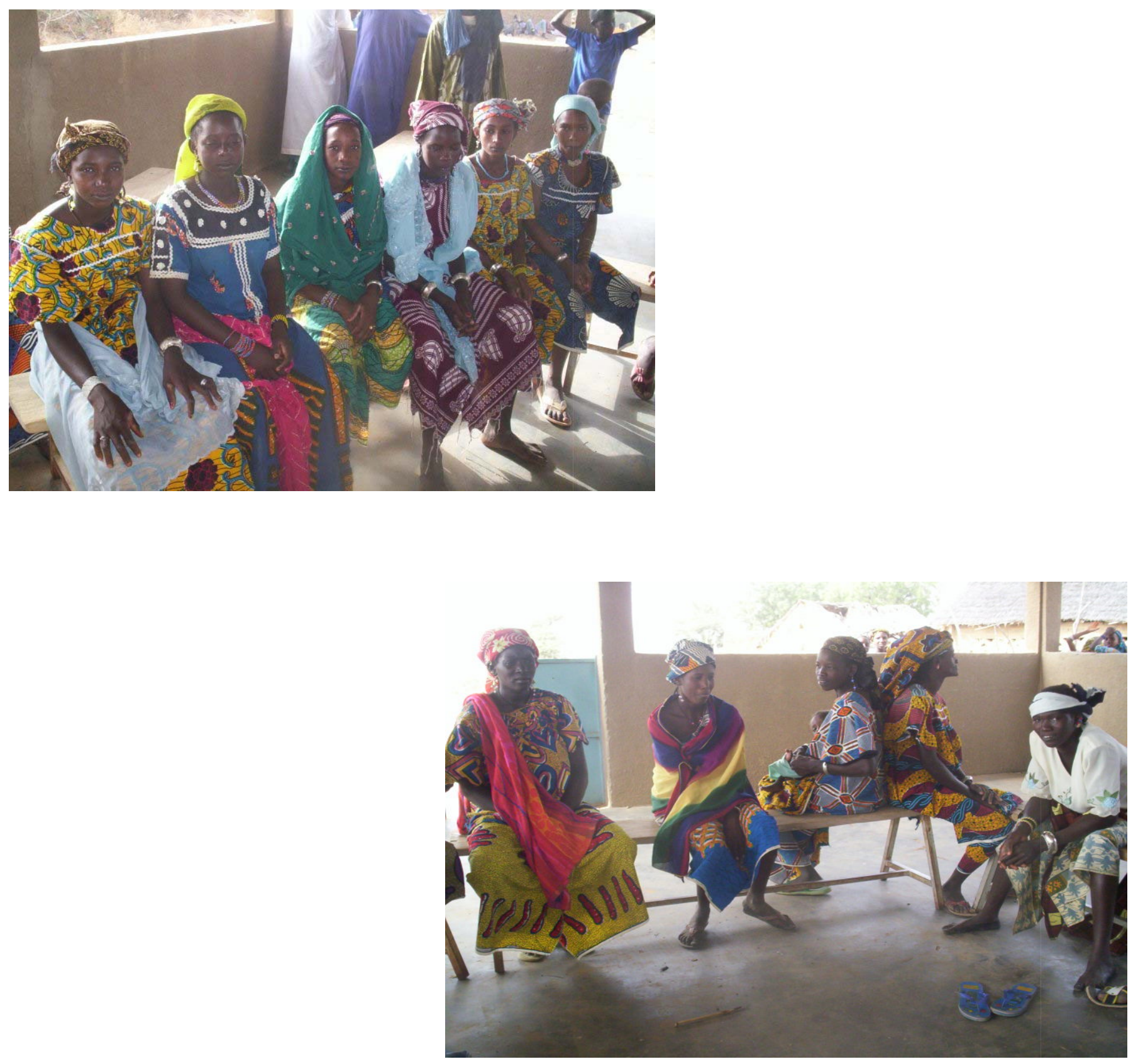

ANALYSE SECONDAIRE DES DONNEES DE L'ANALYSE SITUATIONNELLE DES SERVICES DE SANTE DE LA REPRODUCTION 


\section{PHOTOS DE COUVERTURE}

PHOTO 1: Focus Group avec des adolescentes venues pour la CPN

PHOTO 2: Focus Group avec des adolescentes post-partum dans un centre de santé

\section{EQUIPE DE RECHERCHE}

Population Council

Gisèle Kaboré

\section{Consultant}

Idrissa Kaboré 


\section{SOMMAIRE}

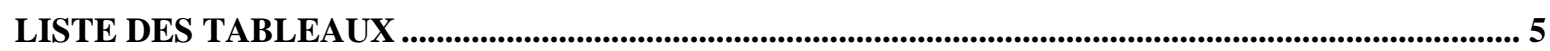

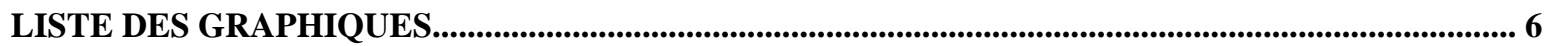

LISTE DES SIGLES ET ABREVIATIONS .............................................................................................. 7

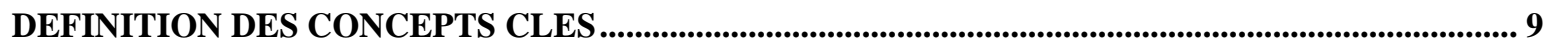

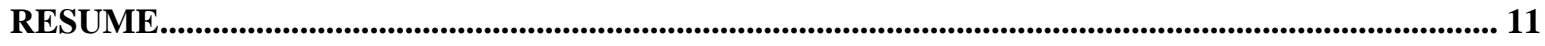

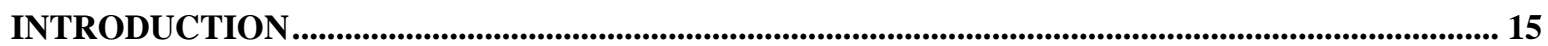

I. PRESENTATION DE L'ANALYSE SECONDAIRE ................................................................................. 15

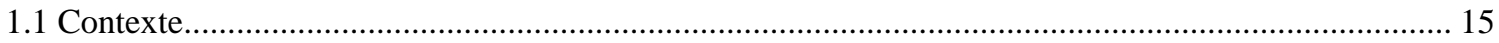

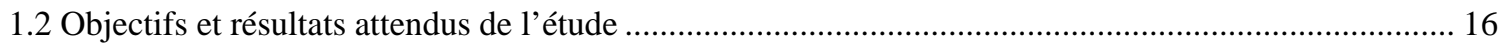

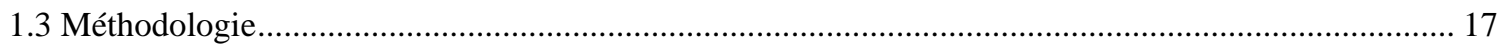

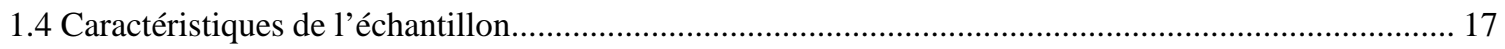

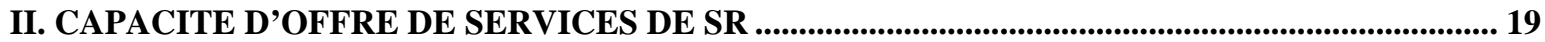

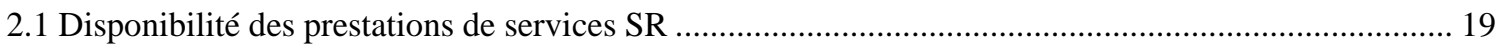

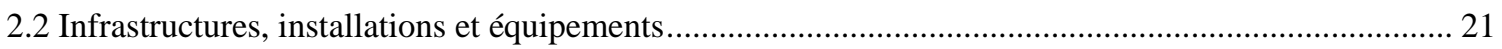

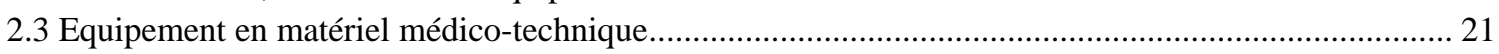

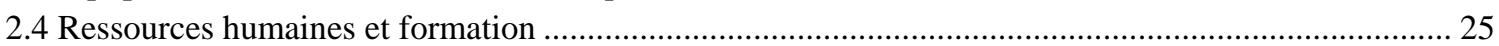

2.5 Disponibilité et utilisation du matériel IEC pour les activités de santé maternelle................................ 28

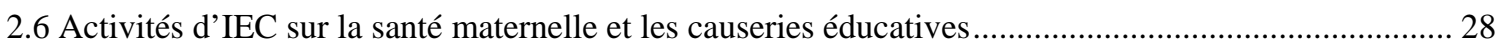

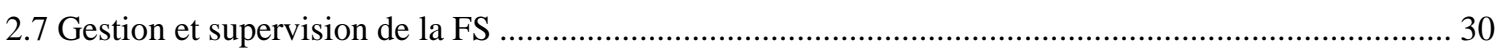

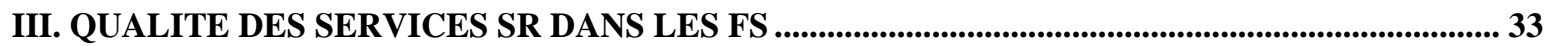

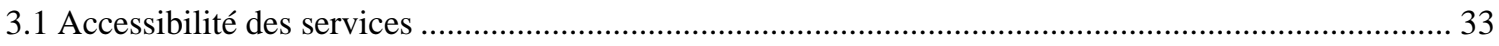

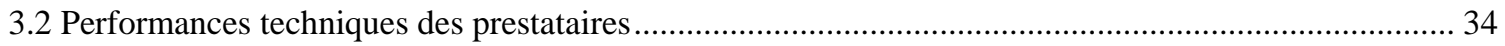

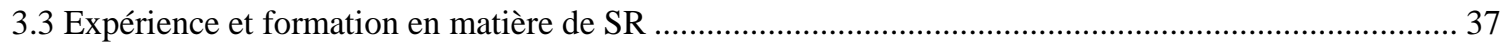

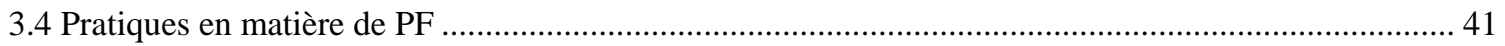

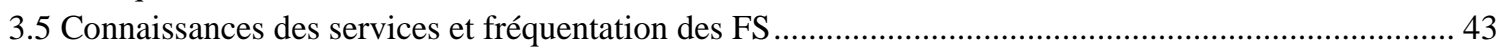

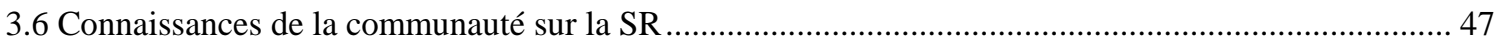

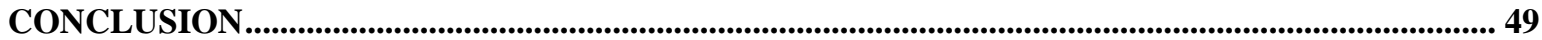

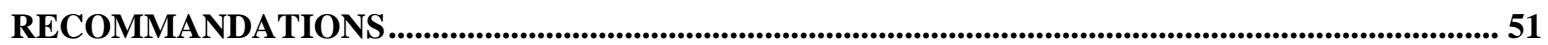

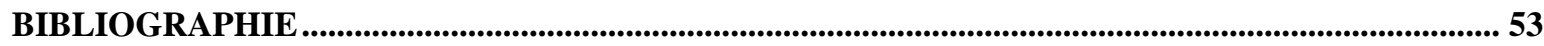





\section{LISTE DES TABLEAUX}

Tableau 1 : Répartition des formations sanitaires de l'échantillon par type et par région ........ 17

Tableau 2 : Caractéristiques de l'échantillon .......................................................................... 18

Tableau 3 : Ages des clients, des femmes et des hommes de la communauté par région ........ 18

Tableau 4 : Répartition des prestations disponibles par région............................................... 20

Tableau 5 : Répartition des FS selon les caractéristiques ou la disponibilité des lieux d'examen par région ..................................................................................... 21

Tableau 6 : Disponibilité du matériel de base dans les formations sanitaires par région

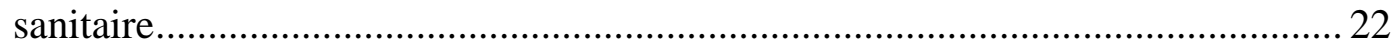

Tableau 7 : Disponibilité des fongibles médicaux essentiels pour les accouchements et les

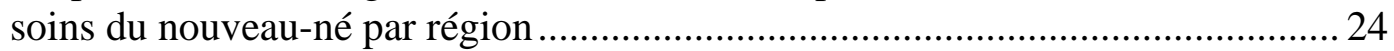

Tableau 8 : Proportion des CSPS selon la disponibilité du personnel .................................... 25

Tableau 9 : Proportion des prestataires qui ont reçu une formation en SR dans les 12 mois ayant précédé l'enquête par région et par thème ............................................... 27

Tableau 10 : Disponibilité et thématiques du matériel IEC pour les prestations de soins de santé maternelle par région............................................................................. 28

Tableau 11 : Proportions de prestataires selon les éléments d’investigation de la consultation en $\mathrm{CPN}$ et en PF.

Tableau 12 : Proportions de prestataires selon les éléments d’investigation de la consultation

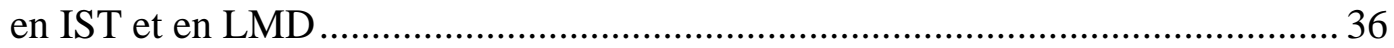

Tableau 13 : Proportions des prestataires observés ayant demandé ou réalisé des tests ou examens paracliniques pendant la consultation de CPN par type de formation sanitaire.

Tableau 14 : Proportions des prestataires par service fournis aux clients et par région sanitaire 38

Tableau 15 : Proportions par service des prestataires ayant reçu des compétences au cours de leur formation de base et de prestataires ayant reçu un recyclage en cours d'emploi par région .

Tableau 16 : Proportions des prestataires selon la méthode fournie les six derniers mois et proportions de clients observés en PF auxquels des méthodes de contraception ont été présentées par type de méthode

Tableau 17 : Proportions des prestataires observant certaines restrictions par méthode contraceptive.

Tableau 18 : Proportion des clients selon la connaissance des services offerts dans les FS par région

Tableau 19 : Proportions des femmes et proportions des hommes selon les facteurs de risque de la grossesse et selon les signes de complications du travail et d'une grossesse 47 


\section{LISTE DES GRAPHIQUES}

Graphique 1 : Proportions de CSPS disposant du matériel minimum requis pour les accouchements par région

Graphique 2 : Proportion des clientes ayant reçu des conseils lors de la consultation par région . 29

Graphique 3 : Proportion des clientes ayant participé à des causeries éducatives le jour de la consultation par région

Graphique 4 : Proportions des FS ayant connu une rupture de stock de médicaments et consommables de SR au cours des 12 derniers mois par région.

Graphique 5 : Proportions de prestataires effectuant des supervisions par région. 31

Graphique 6 : Proportions de prestataires disposant de grille de supervisions par région....... 31

Graphique 7 : Existence de COGES fonctionnel par région .................................................. 32

Graphique 8 : Proportions des clients satisfaits des heures d'ouverture des FS par région..... 33

Graphique 9: Proportions des clients ayant reçu gratuitement le ticket de consultation pour la CPN par région

Graphique 10 Proportions des prestataires par méthode contraceptive qu’ils ne recommanderaient pas pour les adolescents

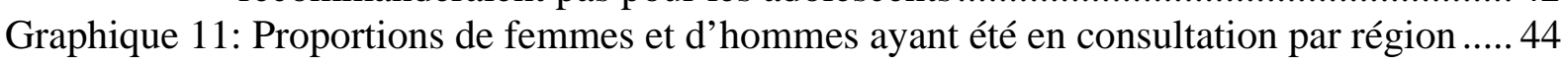

Graphique 12: Proportions des femmes selon l'utilisation des services au cours des 12 derniers mois

Graphique 13: Proportions de femmes et d'hommes ayant été en consultation 45

Graphique 14: Proportions de femmes et d'hommes connaissant et utilisant les services d'ASC 46 


\section{LISTE DES SIGLES ET ABREVIATIONS}

AA

AIS

AV

AMIU

CAMEG

CCC

CDV

CHR

$\mathrm{CHU}$

CIPD

CMA

COGES

CPN

CSPS

DIU

DRD

DSF

EDS

FS

GDT

IB

IDE

IEC

IST

ITG

JNV

LMD

MAMA

MEG

MMR

OMS

ONG

PCIME

PEV

PF

PNDS

PNUD

PSADO

PTME

RAJS/BF

SOU

SONU

SOUB

SR

SRAJ

SRO

UNFPA

ZI
: Accoucheuse Auxiliaire

: Agent Itinérant de Santé

: Accoucheuse Villageoise

: Aspiration Manuelle Intra Utérine

: Centrale d'Achat des Médicaments Essentiels Génériques

: Communication pour le Changement de Comportement

: Centre de Dépistage Volontaire

: Centre Hospitalier Régional

: Centre Hospitalier Universitaire

: Conférence Internationale sur la Population et le Développement

: Centre Médicale avec Antenne Chirurgicale

: Comité de Gestion

: Consultation Prénatale

: Centre de Santé et de Promotion Sociale

: Dispositif Intra Utérin

: Dépôt Répartiteur du District

: Direction de la santé de la Famille

: Enquête Démographique et de Santé

: Formation sanitaire

: Guide Diagnostic et Traitement

: Infirmier Breveté

: Infirmier Diplômé d'Etat

: Information Education Communication

: Infection Sexuellement transmissible

: Inflammation du tractus génital

: Journées Nationales de Vaccination

: Thérapie de réhydratation Orale

: Méthode de l'allaitement maternel et de l'aménorrhée

: Médicaments Essentiels Génériques

: Maternité à Moindre Risque

: Organisation Mondiale de la Santé

: Organisation Non Gouvernementale

: Prise en Charge Intégrée des Maladies de l'Enfant

: Programme Elargi de Vaccination

: Planning Familial

: Plan National de Développement Sanitaire

: Programme des Nations Unies pour le développement

: Projet Santé sexuelle des adolescents

: Prévention Transmission Mère-Enfant

: Réseau Africain de Jeunes contre le Sida / Burkina Faso

: Soins Obstétricaux d'Urgence

: Soins Obstétricaux et Néonataux d’Urgence

: Soins Obstétricaux d’Urgence de Base

: Santé de la Reproduction

: Santé Reproductive des Adolescents et Jeunes

: Sel de Réhydratation Orale

: Fonds des Nations Unies pour la Population

: Zone d'Intervention 



\section{DEFINITION DES CONCEPTS CLES}

Analyse situationnelle : Etude menée dans les formations sanitaires pour rendre disponibles des informations sur la capacité fonctionnelle et la qualité des services. A l'origine, l'analyse situationnelle (ANASIT) réalisée dans plusieurs pays en développement s’intéressait essentiellement à la planification familiale, mais depuis la CIPD tenue au Caire en 1994, les analyses situationnelles prennent en compte la santé de la reproduction de façon générale. La présente ANASIT a la particularité d'inclure le volet communautaire dans la collecte des données. La méthodologie de l’ANASIT repose sur :

- L'interview des prestataires et des clients utilisateurs des services de SR,

- L'observation de l'interaction prestataires/clients,

- L’inventaire du matériel et des équipements.

Les équipes de collecte passent une journée dans chaque formation sanitaire retenue. Cela constitue une limite pour la réalisation des différentes observations et des interviews envisagées, dans la mesure où les activités ne sont pas intégrées dans toutes les formations sanitaires.

Santé de la Reproduction : Selon la CIPD, la SR se définit comme étant le bien-être général, tant physique que mental et social, de la personne humaine, pour tout ce qui concerne l'appareil génital, ses fonctions et son fonctionnement et non pas seulement l'absence de maladies ou d'infirmités. Cela suppose donc qu'une personne peut mener une vie sexuelle satisfaisante en toute sécurité, qu'elle est capable de procréer et libre de le faire aussi souvent et aussi peu souvent qu'elle le désire. Cette dernière condition implique qu'hommes et femmes ont le droit d'être informés et d'utiliser la méthode de planification de leur choix, ainsi que d'autres méthodes de leur choix de régulation des naissances qui ne soient pas contraires à la loi, méthodes qui doivent être sûres, efficaces, abordables et acceptables, ainsi que le droit d'accéder à des services de santé qui permettent aux femmes de mener à bien grossesse et accouchement et donnent aux couples toutes les chances d'avoir un enfant en bonne santé.

\section{Zone d'intervention du projet :}

La zone d'intervention du projet «Eliminer le mariage des enfants au Burkina » est circonscrite dans 24 localités reparties dans 5 régions administratives du Burkina Faso. Elle couvrait $11^{1}$ districts sanitaires (DS) en 2006 dont la répartition est la suivante :

- Centre-nord : DS de Boulsa (06 FS), DS de Kaya (07 FS) ;

- Sahel : DS de Gorom (06 FS), DS de Dori (09 FS) ;

- Centre-est : DS de Tenkodogo (09 FS), DS de Zabré (06 FS), DS de Ouargaye (08 FS) ;

- Centre-sud : DS de Manga (04 FS), DS de Kombissiri (04 FS) ;

- Est : DS de Bogandé (08 FS), DS de Fada (07 FS).

\footnotetext{
${ }^{1}$ En 2008, avec le nouveau découpage, la zone d'intervention du projet s'inscrit dans 13 districts sanitaires (DS).
} 



\section{RESUME}

En début 2002, l'UNFPA a soutenu un projet intitulé Renforcement des services sanitaires et sociaux pour faire face aux besoins de santé sexuelle et reproductive des adolescentes. Il est connu que les filles sont très nettement désavantagées au Burkina Faso et ont beaucoup moins de possibilités en matière d'éducation et de santé. Elles sont plus sujettes au mariage précoce, une pratique traditionnelle néfaste qui perpétue les inégalités de genre au sein de la société.

Le projet avait pour objectif d'améliorer la connaissance des adolescentes mariées en ce qui concerne la législation et leurs propres droits, les éduquer à prendre soin de leur propre santé et de celle de leurs enfants, et augmenter leur accessibilité aux services de santé reproductive, particulièrement dans les régions les plus pauvres du Burkina Faso. Ce sont le Centre-sud, l'Est, le Sahel, le Centre-est et le Centre-nord où les indicateurs de développement sont les plus bas avec une forte proportion de mariages précoces et où plus de la moitié des filles ayant entre 10 et 19 ans est déjà donnée en mariage.

La présente analyse utilise les données de l'analyse situationnelle de 2006. C’est une analyse secondaire qui s'intéresse à la zone d'intervention de l'UNFPA. Elle couvre 11 districts sanitaires rassemblant 12 centres de référence (hôpitaux et CMA), et 62 CSPS. Pour la réalisation de cette analyse, les données de la zone d'intervention ont été extraites de la base de données de l'analyse situationnelle de 2006. Un effectif de 167 prestataires de SR relevant de la zone d'intervention ont été extraits de la base. Parmi les femmes et les hommes dans la communauté, une trentaine d'adolescents est dénombrée dans chaque groupe. Cet effectif est très réduit pour permettre des analyses au niveau des régions. L’analyse globale a donc été privilégiée.

Dans la zone d'intervention, la plupart des prestations sont disponibles. Neuf prestations sont offertes par la totalité des centres de références, il s'agit de l'accouchement, des soins PostPartum (suite de couches), des soins Post abortum, de la consultation postnatale, de la consultation Infécondité/ Infertilité, de l'IEC IST autres que VIH/SIDA, du traitement IST autres que VIH SIDA, des tests VIH/SIDA et du diagnostic laboratoire des IST autres que VIH. Les prestations les moins offertes sont la surveillance nutritionnelle pondérale, la récupération nutritionnelle et la PTME. Dans les CSPS, les prestations les plus offertes sont la contraception, l'IEC/ PF, la consultation prénatale, l'accouchement, les soins post-partum (suite de couches), les soins post-abortum, la consultation postnatale, la vaccination (mère/enfant), l'IEC nutrition, la LMD (thérapie de réhydratation orale) et l'IEC VIH/SIDA. La prise en charge des IST/ SIDA hommes, la PCIME, la prise en charge des IST/SIDA aux adolescents, la PTME, la prise en charge pédiatrique du VIH, les tests VIH/SIDA et le diagnostic laboratoire des IST autres que VIH sont très peu disponibles.

Les caractéristiques des salles d'examen relatives à la discrétion du regard et de l'écoute, l'éclairage, la propreté et l'aération du lieu d'examen sont d'un niveau acceptable. On y dénombre 4 FS sur 5. La propreté et l'éclairage dans les FS font plus défaut au Sahel. Peu de FS disposent de toilettes/latrines fonctionnelles et système d'évacuation des eaux usées.

Dans les CSPS, les équipements de base manquent également. La montre chronomètre/compte-seconde, le stéthoscope obstétrical, le thermomètre médical, le stéthoscope médical, la balance pour peser les adultes, le tensiomètre, le stérilisateur (poupinel, autoclave...), la ventouse obstétricale, le Kit pour (AMIU) et le matériel de 
protection (bottes/sabots, bonnet, bavette, blouse et tabliers) se retrouvent dans 8 FS sur 10 . Les boîtes à instruments particulièrement, le garrot, les pinces obstétricales, les brancards et le kit pour dilatation et curetage font énormément défaut car très peu de FS en disposent. A l'inverse, les tubes et masques d'oxygénations, le matériel de protection (bottes/sabots, bonnet, bavette, blouse et tabliers), le garrot, la ventouse obstétricale, les boites à instrument, le spéculum (diverses tailles) et les pinces obstétricales sont disponibles dans quelques rares CSPS. Les boîtes d'accouchement complet ne sont pas disponibles dans toutes les FS à l'exception de celles du Centre-est. Les différences de la disponibilité du matériel minimum dans les FS sont très variables dans la zone d'intervention. Le Sahel, l'Est et le Centre-sud ont un peu plus de 1 FS sur 4 qui ne disposent pas du matériel minimum pour les accouchements. Le matériel IEC disponible dans les FS pour les soins de santé maternelle est essentiellement composé d'affiches.

Les fongibles médicaux essentiels tels que les gants chirurgicaux et les kits pour perfusion sont disponibles dans toutes les FS. Dans les CSPS, les kits pour perfusion, les seringues et les aiguilles jetables ainsi que les compresses stériles sont disponibles dans la majorité des FS. Cependant les gants de révision utérine, le partographe, les kits d'oxygène, le matériel stérile pour tamponnement utérin ou serviette hygiénique stérile, la trousse de test de la syphilis, les kits pour le test du VIH, les poches de sang et les kits pour transfusion de sang manquent cruellement dans certaines FS.

Plus de la moitié des FS répondent aux normes en matière de ressources humaines c'est-à-dire que la FS dispose d'au moins un infirmier, une accoucheuse auxiliaire et un AIS ou à défaut un manœuvre. Pour 22,6 \% des CSPS, le fonctionnement se fait avec un nombre de prestataires inférieur à 3 agents et il en est autant pour les CSPS avec 3 agents ou plus sans avoir tous les profils d'agents requis. Dans les régions de l'Est, du Centre-nord et du Centresud, moins de la moitié des FS répondent aux normes.

Les protocoles cliniques (guides de diagnostic et traitement, algorithmes/ordinogrammes) sont disponibles dans la plupart des FS. La faible disponibilité des documents de référence s'accompagne de leur faible utilisation (40,4\%). L'utilisation d'outils standardisés pour la supervision n'est pas de règle. Moins d'un prestataire sur 5 dispose d'une grille de supervision. L'affiche reste le matériel IEC le plus disponible dans les FS. Les domaines de la santé qui y sont abordés sont essentiellement la SR.

Des lacunes sont observées dans les procédures de l'interrogatoire et de l'examen physique dans les différents types de services. Les corrections de ces lacunes requièrent une supervision régulière et un recyclage ou une mise à niveau des prestataires. Par rapport aux adolescents, on remarque que seulement $1 / 3$ des prestataires s'intéressent à l'âge des clients, éléments pertinents pour considérer les préoccupations de cette catégorie de population.

La rupture de stock de médicaments a été effective dans 3 FS sur 5. Elle concerne plus les FS du Centre-nord. De nombreux produits sont concernés par les ruptures de stock. Il s'agit de : kit IST, injectables, SRO, pilule, quinine $300 \mathrm{mg}$ cp, Fer + AF $225 \mathrm{mg}$ cp, gants d'examen, Cotrimoxazole 480mg cp, AAS 500mg cp, condoms, paracétamol, chloroquine $100 \mathrm{mg}$ cp, Métronidazole $250 \mathrm{mg}$ cp, Mébendazole et kit césarienne. Le réapprovisionnement se fait dans la majorité des FS (52,7 \%) à intervalles réguliers, ou quand le stock atteint le seuil de sécurité pour 43,2 \% d'entre elles. D’une manière générale, les contraceptifs oraux $(1,4 \%)$ et les injectables (5,4\%) sont non disponibles dans la majorité des FS. 
Les services du paquet minimum d'activités de SR ne sont pas tous offerts. Moins de $50 \%$ des prestataires offrent les services tels que le test VIH/SIDA, la PTME et le diagnostic de laboratoire des IST autres que VIH/SIDA. Les méthodes contraceptives les plus fournies par les prestataires sont les pilules $(93,7 \%)$ et les injectables $(93,0 \%)$. Les autres méthodes sont peu fournies car moins de $50 \%$ des prestataires l'affirment.

Pour plus de la moitié des clients la consultation prénatale, l'accouchement, les services curatifs-adulte et les services curatifs-enfant sont les services connus. Environ 1 femme sur 4 et 3 hommes sur 5 ont été en consultation. Cette fréquentation est variable d'une région à l’autre. La fréquentation la plus faible est observée à l’Est.

Pour plus de la moitié des hommes et des femmes, le jeune âge de la femme enceinte est un facteur de risque tout comme les grossesses rapprochées et les grossesses multiples. Les signes de complication de la grossesse sont par contre moins connus des femmes et des hommes. En général, moins de 1 personne sur 4 connaît ces signes. Les vertiges, la forte fièvre, l'anémie ou la pâleur et les membres ou visage enflés sont souvent cités. Les saignements vaginaux au cours de la grossesse et l'immobilité du fœtus en particulier semblent ne pas constituer un signe d'alerte.

Les douleurs lombaires (des reins et/ou du bas-ventre), sont les signes connus des femmes et des hommes comme éléments annonciateurs du travail. La durée prolongée du travail est un signe important de danger pendant le travail de la femme. Le saignement après l'accouchement et la présentation non céphalique ne sont pas considérés comme très préoccupants car moins de femmes et d’hommes y ont fait référence.

Quelques recommandations ont été formulées en réponses aux problèmes et aux insuffisances constatées, sur la capacité d’offre et la qualité des services. 



\section{INTRODUCTION}

L'analyse secondaire des données de l'analyse situationnelle des services de santé de la reproduction au Burkina s'inscrit dans le cadre de la mise en œuvre du projet «Eliminer le mariage des enfants au Burkina: un plan pour la protection, le renforcement et l'action communautaire ». Elle vise à fournir les bases pour l'appréciation de la disponibilité des services et leur utilisation par les adolescents dans la zone d'intervention du projet. Cette analyse est faite à partir de la base de données de l'Analyse situationnelle des services de SR offerts par les formations sanitaires du Burkina réalisée par le Population Council en 2006. Cela signifie que les informations contenues dans ce rapport rendent compte d'une situation vécue en 2006, d'où la nécessité de tenir compte des possibles améliorations qui ont pu être faites depuis cette période.

Le présent rapport s'intéresse dans un premier temps au contexte, aux objectifs et à la méthodologie de l'étude, puis aux résultats sur la capacité fonctionnelle et la qualité de l'offre des services dans les formations sanitaires (FS), et enfin l'utilisation des services par les communautés.

\section{PRESENTATION DE L’ANALYSE SECONDAIRE}

\subsection{Contexte}

Au Burkina Faso, les filles sont en général très nettement désavantagées et ont moins accès à l'éducation et à la santé. Elles sont plus sujettes au mariage précoce, une pratique traditionnelle néfaste qui perpétue les inégalités de genre au sein de la société, affectant la sécurité humaine des filles et des femmes. Pour y remédier, le MASSN, l'UNFPA et l'UNICEF, ont entrepris un projet multisectoriel pour empêcher les mariages précoces et améliorer les conditions de vie des filles mariées. Le projet se fonde sur des interventions pilotes encourageantes qui ont été menées avec succès par des partenaires en collaboration avec des ONG locales et le Gouvernement du Burkina Faso.

En début 2002, l'UNFPA a soutenu un projet intitulé « Renforcement des services sanitaires et sociaux pour faire face aux besoins de santé sexuelle et reproductive des adolescentes". L'objectif de ce projet était d'améliorer les connaissances des adolescentes mariées en ce qui concerne la législation et leurs propres droits, de les éduquer à prendre soin de leur propre santé et de celle de leurs enfants, et d'augmenter leur accessibilité aux services de santé reproductive. Cette intervention, conçue et formulée par le Population Council et l'UNFPA, a été très probante car les jeunes épouses sont conseillées par des paires éducatrices, elles-mêmes étant de jeunes femmes burkinabés mariées. Ainsi de façon tangible, les adolescentes mariées dans les provinces du Bazèga et du Gourma ont reçu pour la première fois, et surtout dans leur propre domicile, des informations acceptables par leurs belles familles, et cela offre un soutien social extra familial aux filles. Il a été démontré que cette intervention puisse s'étendre aux filles mariées vulnérables dans les régions les plus pauvres du Burkina Faso.

Dans la même période, l'UNICEF a entrepris une intervention pour un changement de comportement dans le domaine de la communication à travers le Plan Intégré de Communication (PIC), pour promouvoir le Code des Personnes et de la Famille. 
Dans un autre effort, l'UNICEF a collaboré avec le Forum des Femmes Africaines Educatrices/Burkina Faso (FAWE), une ONG locale, pour établir un programme de lutte contre le trafic des enfants, la réhabilitation des adolescentes victimes du trafic des enfants et leurs mères.

L'amélioration des indicateurs d'utilisation des services de la SR passe en grande partie par une double disponibilité des services de SR et d'informations de qualité accessibles à tous. Les services de SR appropriés sont ceux-là qui répondent à certaines normes de quantité et de qualité.

Amener les bénéficiaires à utiliser les services de SR est une tâche plus délicate en raison entre autres, de la méconnaissance qu'ont les populations des services et des barrières socioculturelles.

\subsection{Objectifs et résultats attendus de l'étude}

L'objectif de cette analyse secondaire est d'évaluer la qualité des services de SR offerts aux adolescents et aux jeunes dans la zone d'intervention du projet (24 localités) en vue d'orienter le projet dans ces efforts d'amélioration des services pour les adolescentes.

De façon spécifique, il s’agit :

1. de recueillir des informations sur l'offre et la qualité des services de la santé de la reproduction dans la zone d'intervention du projet;

2. d’apprécier le fonctionnement des services de santé de la reproduction dans la zone d'intervention du projet ;

3. d'apprécier l'utilisation des services par les bénéficiaires et de façon spécifique par les adolescentes;

4. d'identifier les différents problèmes entravant la mise en œuvre des activités de santé de la reproduction dans la zone d'intervention du projet ;

5. de proposer des stratégies pour une meilleure collecte des données, planification, mise en œuvre et suivi des activités de Santé de la Reproduction dans les régions et districts de la zone d'intervention du projet ;

6. de faire des recommandations pour l'amélioration des services SRAJ offerts les formations sanitaires de la zone d'intervention.

\section{- Résultats attendus}

- Des informations sur l'offre et la qualité des services de la santé de la reproduction dans la zone d'intervention sont disponibles ;

- Des informations sur le fonctionnement des services de santé de la reproduction sont disponibles ;

- Des informations sur l'utilisation des services de la santé de la reproduction par les bénéficiaires surtout par les adolescentes sont disponibles ;

- Les différents problèmes entravant la mise en œuvre des activités de santé de la reproduction sont identifiés.

- Des stratégies pour une meilleure collecte des données et de suivi des activités de Santé de la Reproduction dans les régions et districts de la zone d'intervention du projet existent. 
Des recommandations pour l'amélioration des services SRAJ offerts par les formations sanitaires de la zone d’intervention sont formulées.

\subsection{Méthodologie}

Pour la réalisation de cette analyse, les données de la zone d’intervention ont été extraites de la base de données de l'analyse situationnelle des services de santé de la reproduction réalisée en 2006. C'est une analyse secondaire qui s'intéresse à la zone d'intervention du projet « éliminer le mariage des enfants au Burkina ». Initialement le projet est exécuté dans 24 localités reparties dans 5 régions administratives du Burkina. Parmi les 24 localités de la zone d'intervention du projet, des formations sanitaires (FS) de seize (16) d'entre elles ont été couvertes par l'analyse situationnelle de 2006.

$\underline{\text { Tableau } 1 \text { : Répartition des formations sanitaires de l'échantillon par type et par région }}$

\begin{tabular}{lcccccc}
\hline \multicolumn{1}{c}{ Types de FS } & Centre-nord & Sahel & Centre-est & Centre-sud & Est & Ensemble \\
\hline CHR & 1 & 1 & 1 & 0 & 1 & 4 \\
CMA & 1 & 2 & 2 & 2 & 1 & 8 \\
CSPS & 11 & 12 & 20 & 6 & 13 & 62 \\
\hline Ensemble des FS & 13 & 15 & 23 & 8 & 15 & 74 \\
\hline Nombre de districts sanitaires & 2 & 2 & 3 & 2 & 2 & 11 \\
\hline
\end{tabular}

Au regard de la faiblesse des échantillons touchés dans ces zones, nous avons trouvé judicieux de considérer les districts sanitaires et non les formations sanitaires de façon spécifique. Ceci a l'avantage de renseigner des localités dont les formations sanitaires n’ont pas été ciblées au moment de l'analyse situationnelle de 2006. Aussi, dans les zones urbaines, nous avons pris en compte la totalité des formations sanitaires qui ont fait l'objet d'enquête. En effet, la présente analyse secondaire concerne au total 74 formations sanitaires dont 12 centres de référence (hôpitaux et CMA) et 62 CSPS (Tableau 1). Ces formations sanitaires sont reparties dans 11 districts sanitaires (DS) dont la répartition est la suivante :

- Centre-nord : DS de Boulsa (06 FS), DS de Kaya (07 FS) ;

- Sahel : DS de Gorom (06 FS), DS de Dori (09 FS) ;

- Centre-est : DS de Tenkodogo (09 FS), DS de Zabré (06 FS), DS de Ouargaye (08 FS);

- Centre-sud : DS de Manga (04 FS), DS de Kombissiri (04 FS) ;

- Est : DS de Bogandé (08 FS), DS de Fada (07 FS).

Au regard des effectifs par région, il ne s'avérait pas indiqué d'envisager l'analyse par district sanitaire, en ce sens que les effectifs étaient souvent très réduits. Le choix d'un niveau plus agrégé s’imposait. Ainsi selon le cas, les résultats sont produits pour l'ensemble de la zone d'intervention ou par région. La région reste ici un regroupement de FS pour l'analyse et non une réalité administrative unique.

\subsection{Caractéristiques de l'échantillon}

\section{$\checkmark$ Les prestataires de santé}

A partir de la base de données disponible, un total de 167 prestataires de SR relevant de la zone d'intervention du projet ont été extraits. La répartition par catégorie de personnel a donné 51 accoucheuses auxiliaires, 44 infirmiers d'Etat, 24 sages-femmes d'Etat ou maïeuticiens, 22 infirmiers brevetés, 18 agents itinérants de santé, 01 médecin généraliste, 02 médecins gynécologues, 01 attaché ou assistant de santé et 04 matrones. Dans les 74 FS 
retenues pour l'analyse dans la zone d'intervention, différents types d'interactions ont été inégalement observés (Tableau 2).

Tableau 2 : Caractéristiques de l'échantillon

\begin{tabular}{lcccccc}
\hline \multicolumn{1}{c}{ Type de prestataires } & Centre-nord & Sahel & Centre-est & Centre-sud & Est & Ensemble \\
\hline Médecin généraliste & & & & & & \\
Médecin gynécologue & 1 & 0 & 0 & 0 & 0 & 1 \\
Attaché, assistant de santé & 1 & 0 & 0 & 0 & 1 & 2 \\
Infirmier(e) d'état & 9 & 0 & 0 & 0 & 0 & 1 \\
Sage-femme d'état Maïeuticien & 3 & 5 & 14 & 3 & 5 & 44 \\
Infirmier Breveté & 4 & 4 & 8 & 0 & 8 & 24 \\
Agent Itinérant de Santé & 3 & 3 & 3 & 1 & 5 & 22 \\
Accoucheuse Auxiliaire & 6 & 7 & 21 & 4 & 5 & 18 \\
Matrones & 1 & 1 & 0 & 2 & 9 & 51 \\
Total & 29 & 34 & 53 & 18 & 33 & 167 \\
\hline Type d'observations & & & & & & 7 \\
\hline PF & 12 & 18 & 19 & 7 & 20 & 76 \\
CPN & 15 & 34 & 50 & 34 & 42 & 175 \\
IST & 2 & 7 & 4 & 3 & 12 & 28 \\
LMD & 5 & 2 & 14 & 1 & 13 & 35 \\
\hline
\end{tabular}

\section{Les clients}

Dans la zone d'intervention, 227 clients des services de santé de la reproduction ont été inventoriés. Dans l'ensemble, les clients sont mariés. L'âge moyen des clients est de 25,7 ans avec des extrêmes allant de 12 à 51 ans et la moitié des clients a moins de 25 ans.

Les clients sont en général sans instruction. On trouve autant de clients alphabétisés $(1 / 10)$, que de clients instruits de niveau primaire $(1 / 10)$ et aussi de niveau secondaire et plus $(1 / 10)$. $\mathrm{Au}$ total, 245 femmes et 238 hommes dans les ménages ont été dénombrés pour l'analyse secondaire dans la zone d'intervention. L'âge moyen des femmes enquêtées se situe à 29,4 ans tandis que celui des hommes est de 35,3 ans (Tableau 3).

Tableau 3 : Ages des clients, des femmes et des hommes de la communauté par région

\begin{tabular}{|c|c|c|c|c|c|c|}
\hline Age & Centre-nord & Sahel & Centre-est & Centre-sud & Est & Ensemble \\
\hline \multicolumn{7}{|l|}{ Clients } \\
\hline Moyen & 26,8 & 24,7 & 25,2 & 23,2 & 28,2 & 25,7 \\
\hline Médian & 26,0 & 24,0 & 25,0 & 21,0 & 28,5 & 25,0 \\
\hline Minimum & 17 & 15 & 15 & 12 & 16 & 12 \\
\hline Maximum & 47 & 51 & 44 & 42 & 47 & 51 \\
\hline Effectif total de clients & 27 & 39 & 66 & 41 & 54 & 227 \\
\hline$\%$ adolescents & 18,5 & 24,4 & 15,1 & 26,8 & 10,0 & 17,8 \\
\hline \multicolumn{7}{|l|}{ Femmes } \\
\hline Moyen & 27,8 & 29,6 & 28,0 & 32,8 & 30,3 & 29,4 \\
\hline Médian & 25,0 & 28,0 & 27,0 & 32,0 & 30,0 & 28,0 \\
\hline Effectif Femmes & 37 & 43 & 79 & 30 & 56 & 245 \\
\hline$\%$ adolescentes & 13,5 & 14,0 & 15,2 & 3,3 & 8,9 & 11,8 \\
\hline \multicolumn{7}{|l|}{ Hommes } \\
\hline Moyen & 33,2 & 36,9 & 34,1 & 39,6 & 35,3 & 35,3 \\
\hline Médian & 30,0 & 34,0 & 35,0 & 39,5 & 34,0 & 35,0 \\
\hline Effectif Hommes & 41 & 47 & 78 & 24 & 48 & 238 \\
\hline$\%$ adolescents & 22,0 & 10,6 & 9,0 & 8,3 & 14,6 & 12,6 \\
\hline
\end{tabular}




\section{CAPACITE D'OFFRE DE SERVICES DE SR}

La capacité d'offre de services est appréciée afin de donner des orientations pour la réussite d'un programme de santé. C'est l'offre de services de qualité qui requiert au minimum i) une disponibilité des services, ii) un cadre approprié, iii) des ressources nécessaires (humaines et matérielles) et une bonne organisation des services.

\subsection{Disponibilité des prestations de services SR}

La plupart des prestations sont disponibles dans la zone d'intervention. On constate qu'il n'y a que neuf (9) prestations qui sont offertes par la totalité des centres de références. Il s’agit de l'accouchement, des soins post-partum (suite de couches), des soins post-abortum, de la consultation postnatale, de la consultation Infécondité/ Infertilité, de l'IEC IST autres que VIH/SIDA, du traitement IST autres que VIH/SIDA, des tests VIH/SIDA et du diagnostic laboratoire des IST autres que VIH (Tableau 4). Les prestations les moins offertes sont la surveillance nutritionnelle pondérale, la récupération nutritionnelle et la PTME.

Dans les Centres de Santé et de Promotion Sociale (CSPS), les prestations les plus offertes sont la contraception, l'IEC/ PF, la consultation prénatale, l'accouchement, les soins postpartum (suite de couches), les soins post-abortum, la consultation postnatale, la vaccination (mère/enfant), l'IEC nutrition, la LMD (thérapie de réhydratation orale) et l'IEC VIH/SIDA. Par contre, la prise en charge des IST/SIDA hommes, la PCIME, la prise en charge des IST/SIDA aux adolescents, la PTME, la prise en charge pédiatrique du VIH, les tests VIH/SIDA et le diagnostic laboratoire des IST autres que VIH sont très peu disponibles (Tableau 4). On remarquera que les prestations destinées spécifiquement aux adolescents (Services PF aux adolescents et prise en charge des IST/SIDA aux adolescents) ne sont pas partout présentes (64,5\%). 


\section{Tableau 4 : Répartition des prestations disponibles par région}

\begin{tabular}{|c|c|c|c|c|c|c|c|c|c|c|c|c|}
\hline \multirow[b]{2}{*}{ Types de services } & \multicolumn{6}{|c|}{ CHR/CMA } & \multicolumn{6}{|c|}{ CSPS } \\
\hline & $\begin{array}{l}\text { Centre- } \\
\text { nord }\end{array}$ & Sahel & $\begin{array}{c}\text { Centre- } \\
\text { est }\end{array}$ & $\begin{array}{l}\text { Centre- } \\
\text { sud }\end{array}$ & Est & Ensemble & $\begin{array}{l}\text { Centre- } \\
\text { nord }\end{array}$ & Sahel & $\begin{array}{c}\text { Centre- } \\
\text { est }\end{array}$ & \begin{tabular}{|c|}
$\begin{array}{c}\text { Centre- } \\
\text { sud }\end{array}$ \\
\end{tabular} & Est & Ensemble \\
\hline Contraception & 2 & 3 & 3 & 2 & 1 & 11 & 11 & 12 & 20 & 6 & 13 & 62 \\
\hline IEC/ PF & 2 & 3 & 3 & 2 & 1 & 11 & 11 & 12 & 20 & 6 & 13 & 62 \\
\hline Consultation Prénatale & 2 & 3 & 3 & 2 & 1 & 11 & 11 & 12 & 20 & 6 & 13 & 62 \\
\hline Accouchement & 2 & 3 & 3 & 2 & 2 & 12 & 11 & 12 & 20 & 6 & 13 & 62 \\
\hline Soins Post-Partum (suite de couches) & 2 & 3 & 3 & 2 & 2 & 12 & 11 & 12 & 20 & 6 & 13 & 62 \\
\hline Soins Post abortum & 2 & 3 & 3 & 2 & 2 & 12 & 11 & 12 & 20 & 6 & 13 & 62 \\
\hline Consultation postnatale & 2 & 3 & 3 & 2 & 2 & 12 & 11 & 12 & 20 & 6 & 13 & 62 \\
\hline Vaccination (mère/enfant) & 2 & 3 & 2 & 2 & 0 & 9 & 11 & 12 & 20 & 6 & 13 & 62 \\
\hline IEC Nutrition & 2 & 2 & 3 & 2 & 1 & 10 & 11 & 12 & 20 & 6 & 13 & 62 \\
\hline LMD (thérapie de réhydratation Orale) & 2 & 2 & 3 & 2 & 2 & 11 & 11 & 12 & 20 & 6 & 13 & 62 \\
\hline IEC VIH/SIDA & 2 & 2 & 3 & 2 & 2 & 11 & 11 & 12 & 20 & 6 & 13 & 62 \\
\hline Surveillance Nutritionnelle Pondérale & 1 & 2 & 3 & 1 & 1 & 8 & 10 & 12 & 20 & 6 & 13 & 61 \\
\hline Consultation Infécondité/ Infertilité & 2 & 3 & 3 & 2 & 2 & 12 & 11 & 10 & 20 & 6 & 12 & 59 \\
\hline IEC IST autres que VIH/SIDA & 2 & 3 & 3 & 2 & 2 & 12 & 9 & 12 & 18 & 6 & 12 & 57 \\
\hline Traitement IST autres que VIH SIDA & 2 & 3 & 3 & 2 & 2 & 12 & 10 & 12 & 17 & 6 & 12 & 57 \\
\hline Consultation du nourrisson & 2 & 3 & 2 & 2 & 1 & 10 & 11 & 12 & 19 & 6 & 9 & 57 \\
\hline Autres soins curatifs -adulte & 2 & 3 & 3 & 2 & 1 & 11 & 11 & 12 & 19 & 6 & 8 & 56 \\
\hline Autres soins curatifs enfants & 2 & 3 & 3 & 2 & 1 & 11 & 11 & 12 & 19 & 6 & 7 & 55 \\
\hline Consultation pour troubles sexualité masculine & 2 & 2 & 3 & 2 & 2 & 11 & 11 & 8 & 19 & 5 & 12 & 55 \\
\hline $\begin{array}{l}\text { Consultation pour incontinence urinaire chez la } \\
\text { femme }\end{array}$ & 2 & 2 & 3 & 2 & 2 & 11 & 10 & 6 & 19 & 5 & 12 & 52 \\
\hline Services PF aux adolescents & 2 & 1 & 3 & 2 & 1 & 9 & 8 & 7 & 20 & 4 & 10 & 49 \\
\hline Récupération Nutritionnelle & 1 & 1 & 3 & 0 & 1 & 6 & 5 & 11 & 20 & 0 & 11 & 47 \\
\hline prestation de PF aux hommes & 2 & 1 & 3 & 2 & 1 & 9 & 6 & 6 & 20 & 2 & 10 & 44 \\
\hline Prise en charge des IST/ SIDA femmes & 2 & 2 & 3 & 2 & 2 & 11 & 0 & 9 & 19 & 6 & 9 & 43 \\
\hline Prise en charge des IST/ SIDA hommes & 2 & 2 & 3 & 2 & 2 & 11 & 0 & 8 & 19 & 6 & 8 & 41 \\
\hline PCIME & 2 & 3 & 2 & 2 & 0 & 9 & 3 & 9 & 18 & 4 & 7 & 41 \\
\hline Prise en charge des IST/SIDA aux adolescents & 2 & 2 & 3 & 2 & 2 & 11 & 0 & 7 & 19 & 6 & 8 & 40 \\
\hline PTME & 2 & 1 & 2 & 0 & 1 & 6 & 4 & 3 & 10 & 0 & 5 & 22 \\
\hline Prise en charge pédiatrique du VIH & 2 & 1 & 3 & 2 & 2 & 10 & 2 & 1 & 6 & 0 & 1 & 10 \\
\hline Tests VIH/SIDA & 2 & 3 & 3 & 2 & 2 & 12 & 1 & 2 & 2 & 0 & 2 & 7 \\
\hline Diagnostic laboratoire des IST autres que VIH & 2 & 3 & 3 & 2 & 2 & 12 & 2 & 0 & 2 & 0 & 1 & 5 \\
\hline Total des FS & 2 & 3 & 3 & 2 & 2 & 12 & 11 & 12 & 20 & 6 & 13 & 62 \\
\hline
\end{tabular}




\subsection{Infrastructures, installations et équipements}

Une salle d'accouchement fonctionnelle est présente dans plus de la moitié des FS (51 FS sur 74). Pour les FS qui ne disposent pas de salle d'accouchement fonctionnelle, il s'agit en fait de l'absence d'un espace destiné spécifiquement aux accouchements. Dans la réalité, les accouchements et les autres services sont menés au même endroit.

Les commodités indispensables pour une bonne offre de service prennent en compte la discrétion, l'éclairage, la propreté, l'aération, les toilettes ou latrines, l'eau, le traitement des eaux usées et des déchets ainsi que les équipements médicaux nécessaires. Les caractéristiques des salles d'examen relatives à la discrétion du regard et de l'écoute, l'éclairage, la propreté et l'aération du lieu d'examen sont d'un niveau acceptable car 4 FS sur 5 en sont concernées (Tableau 5). La propreté et l'éclairage dans les FS font plus défaut au Sahel. Les différences observées entre les régions ne sont cependant pas significatives au regard des $\mathrm{Ki}^{2}$. Il n'y a donc pas de variabilité nette.

Tableau 5 : Répartition des FS selon les caractéristiques ou la disponibilité des lieux d'examen par région

\begin{tabular}{lcccccc}
\hline Caractéristiques/Disponibilité & Centre-nord & Sahel & Centre-est & Centre-sud & Est & Ensemble \\
\hline Lieu d'examen est discret des regards & 12 & 15 & 20 & 8 & 15 & 70 \\
Lieu d'examen est discret d'écoute & 11 & 13 & 20 & 8 & 15 & 67 \\
Lieu d'examen éclairé & 13 & 11 & 18 & 5 & 12 & 59 \\
Lieu d'examen propre & 8 & 12 & 22 & 4 & 13 & 59 \\
Lieu d'examen aéré & 12 & 12 & 15 & 6 & 14 & 59 \\
Système d'évacuation des eaux usées & 0 & 6 & 12 & 4 & 6 & 28 \\
Système de traitement des déchets & 3 & 7 & 6 & 2 & 4 & 22 \\
Table et tabouret pour examens gynécologiques & 0 & 1 & 2 & 2 & 2 & 7 \\
Eau & 0 & 2 & 2 & 0 & 2 & 6 \\
Toilettes/Latrines & 0 & 0 & 1 & 0 & 2 & 3 \\
\hline Effectif de FS & 13 & 15 & 23 & 8 & 15 & 74 \\
\hline Présence de salle d'accouchement & 10 & 10 & 17 & 4 & 10 & 51 \\
\hline
\end{tabular}

La disponibilité des installations et équipements relatifs au système d'évacuation des eaux usées et au système de traitement des déchets fait énormément défaut. Il y a moins de 1/3 des FS qui en disposent. Cela est très prononcé pour la table et tabouret pour examens gynécologiques, l'eau et les toilettes ou latrines qui sont pratiquement absents.

\subsection{Equipement en matériel médico-technique}

\section{Matériel de base}

Dans les centres de références, à l'exception de la balance pèse-bébé, la disponibilité des équipements de base n'est pas partout effective. Dans au moins 8 FS sur 10, on retrouve le matériel suivant: la montre chronomètre/compte-seconde, le stéthoscope obstétrical, le thermomètre médical, le stéthoscope médical, la balance pour peser les adultes, le tensiomètre, le stérilisateur (poupinel, autoclave...), la ventouse obstétricale, le kit pour (AMIU) et le matériel de protection (bottes/sabots, bonnet, bavette, blouse et tabliers). Les boîtes à instrument particulièrement, le garrot, les pinces obstétricales, les brancards et le kit pour dilatation et curetage font énormément défaut car très peu de FS en disposent. 


\section{Tableau 6 : Disponibilité du matériel de base dans les formations sanitaires par région sanitaire}

\begin{tabular}{|c|c|c|c|c|c|c|c|c|c|c|c|c|}
\hline \multirow[b]{2}{*}{ Equipement de base } & \multicolumn{6}{|c|}{ CHR/CMA } & \multicolumn{6}{|c|}{ CSPS } \\
\hline & $\begin{array}{l}\text { Centre- } \\
\text { nord }\end{array}$ & Sahel & $\begin{array}{l}\text { Centre- } \\
\text { est }\end{array}$ & $\begin{array}{l}\text { Centre- } \\
\text { sud }\end{array}$ & Est & Ensemble & $\begin{array}{l}\text { Centre- } \\
\text { nord }\end{array}$ & Sahel & $\begin{array}{l}\text { Centre- } \\
\text { est }\end{array}$ & $\begin{array}{l}\text { Centre- } \\
\text { sud }\end{array}$ & Est & Ensemble \\
\hline Balance pour peser les adultes & 2 & 3 & 3 & 1 & 2 & 11 & 11 & 11 & 20 & 6 & 13 & 61 \\
\hline Tensiomètre & 2 & 3 & 3 & 1 & 2 & 11 & 11 & 11 & 20 & 6 & 12 & 60 \\
\hline Thermomètre médical & 2 & 3 & 3 & 1 & 2 & 11 & 11 & 10 & 20 & 4 & 13 & 58 \\
\hline Mètre- Ruban & 2 & 3 & 2 & 1 & 2 & 10 & 11 & 10 & 19 & 5 & 13 & 58 \\
\hline Stéthoscope médical & 2 & 3 & 2 & 2 & 2 & 11 & 10 & 11 & 19 & 4 & 13 & 57 \\
\hline Stérilisateur (poupinel, autoclave...) & 2 & 3 & 3 & 1 & 2 & 11 & 11 & 10 & 20 & 5 & 11 & 57 \\
\hline Montre chronomètre/compte-seconde & 2 & 3 & 3 & 2 & 2 & 12 & 10 & 11 & 19 & 4 & 12 & 56 \\
\hline Stéthoscope obstétrical & 2 & 3 & 3 & 1 & 2 & 11 & 10 & 10 & 18 & 4 & 12 & 54 \\
\hline Sonde rectiligne & 1 & 3 & 3 & 1 & 2 & 10 & 8 & 10 & 18 & 5 & 11 & 52 \\
\hline Sondes de Foley et poches à urine & 2 & 2 & 2 & 2 & 2 & 10 & 9 & 8 & 18 & 5 & 11 & 51 \\
\hline Kit pour (AMIU) & 2 & 3 & 3 & 2 & 1 & 11 & 7 & 10 & 20 & 5 & 6 & 48 \\
\hline Balance pèse bébé & 2 & 3 & 3 & 2 & 2 & 12 & 11 & 6 & 11 & 3 & 10 & 41 \\
\hline Kit pour dilatation et curetage & 1 & 3 & 3 & 0 & 1 & 8 & 5 & 4 & 19 & 5 & 5 & 38 \\
\hline Brancard & 2 & 1 & 2 & 0 & 2 & 7 & 2 & 8 & 12 & 0 & 9 & 31 \\
\hline Tubes et masques d'oxygénations & 2 & 3 & 3 & 1 & 0 & 9 & 2 & 8 & 7 & 1 & 2 & 20 \\
\hline $\begin{array}{l}\text { Matériel de protection (bottes/sabots, } \\
\text { bonnet, bavette, blouse et tabliers) }\end{array}$ & 2 & 2 & 3 & 2 & 2 & 11 & 1 & 3 & 9 & 0 & 5 & 18 \\
\hline Garrot & 0 & 0 & 1 & 0 & 1 & 2 & 1 & 1 & 5 & 2 & 2 & 11 \\
\hline Ventouse obstétricale & 2 & 3 & 2 & 2 & 2 & 11 & 0 & 1 & 1 & 0 & 0 & 2 \\
\hline Boîtes à instruments & 0 & 0 & 0 & 0 & 1 & 1 & 0 & 0 & 1 & 1 & 0 & 2 \\
\hline Spéculum (diverses tailles) & 2 & 3 & 3 & 0 & 1 & 9 & 1 & 0 & 0 & 0 & 0 & 1 \\
\hline Pinces obstétricales & 0 & 2 & 2 & 0 & 2 & 6 & 0 & 0 & 0 & 0 & 0 & 0 \\
\hline Effectif des FS & 2 & 3 & 3 & 2 & 2 & 12 & 11 & 12 & 20 & 6 & 13 & 62 \\
\hline
\end{tabular}


Dans les CSPS, les équipements de base manquent également. Il est important de remarquer qu'au moins $10 \%$ des FS ne disposent pas du matériel de base pour offrir un service de qualité surtout dans la prise en charge des accouchements. La balance pour peser les adultes, le tensiomètre, le thermomètre médical, le mètre-ruban et le stéthoscope médical sont presque partout disponibles. A l'inverse, les tubes et masques d'oxygénations, le matériel de protection (bottes/sabots, bonnet, bavette, blouse et tabliers), le garrot, la ventouse obstétricale, les boîtes à instrument, le spéculum (diverses tailles) et les pinces obstétricales sont disponibles dans très peu de CSPS (Tableau 6).

\section{Matériel minimum absolu requis pour les accouchements}

Le matériel minimum absolu requis pour les accouchements est composé de boîte d'accouchement (ciseaux pour cordon, pinces à cordon, sonde vésicale). Le projet s’inscrivant dans la promotion de la santé sexuelle et reproductive des adolescents, il était important de s'assurer de l'effectivité du matériel minimum requis pour les accouchements car les adolescentes sont concernées par le sujet. Les boîtes d'accouchement complètes ne sont pas disponibles dans toutes les FS à l'exception de celles du Centre-est (Graphique 1). Les différences de la disponibilité du matériel minimum dans les FS sont très variables et statistiquement très significatives $(\mathrm{P}=0,007)$ dans la zone d'intervention. Dans le Sahel, l'Est et le Centre-sud, il y a un peu plus de 1 FS sur 4 qui ne disposent pas du matériel minimum pour les accouchements. Il s'en suit que dans les aires couvertes par ces FS, les adolescents seront obligés de parcourir des distances très longues pour bénéficier de ces services. Ce qui n’est pas sans conséquence.

Graphique 1 : Proportions de CSPS disposant du matériel minimum requis pour les accouchements par région

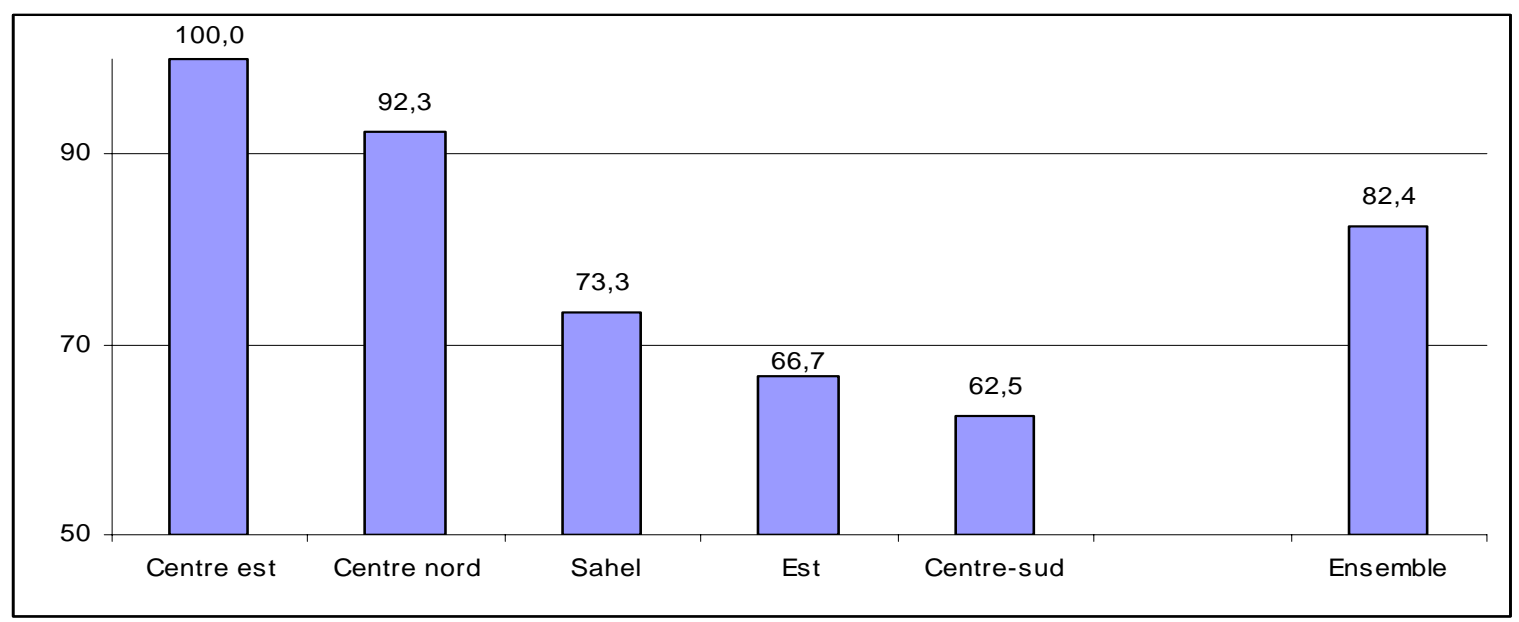

\section{Fongibles médicaux essentiels}

Les fongibles médicaux essentiels tels que les gants chirurgicaux et les kits pour perfusion sont disponibles dans toutes les FS (Tableau 7). Dans les CSPS, les kits pour perfusion, les seringues et les aiguilles jetables ainsi que les compresses stériles sont disponibles dans la majorité des FS. On note cependant que les gants de révision utérine, le partographe, les kits d’oxygène, le matériel stérile pour tamponnement utérin ou serviette hygiénique stérile, la trousse de test de la syphilis, les kits pour le test du VIH, les poches de sang et les kits pour transfusion de sang manquent cruellement dans certaines FS. 
Tableau 7 : Disponibilité des fongibles médicaux essentiels pour les accouchements et les soins du nouveau-né par région

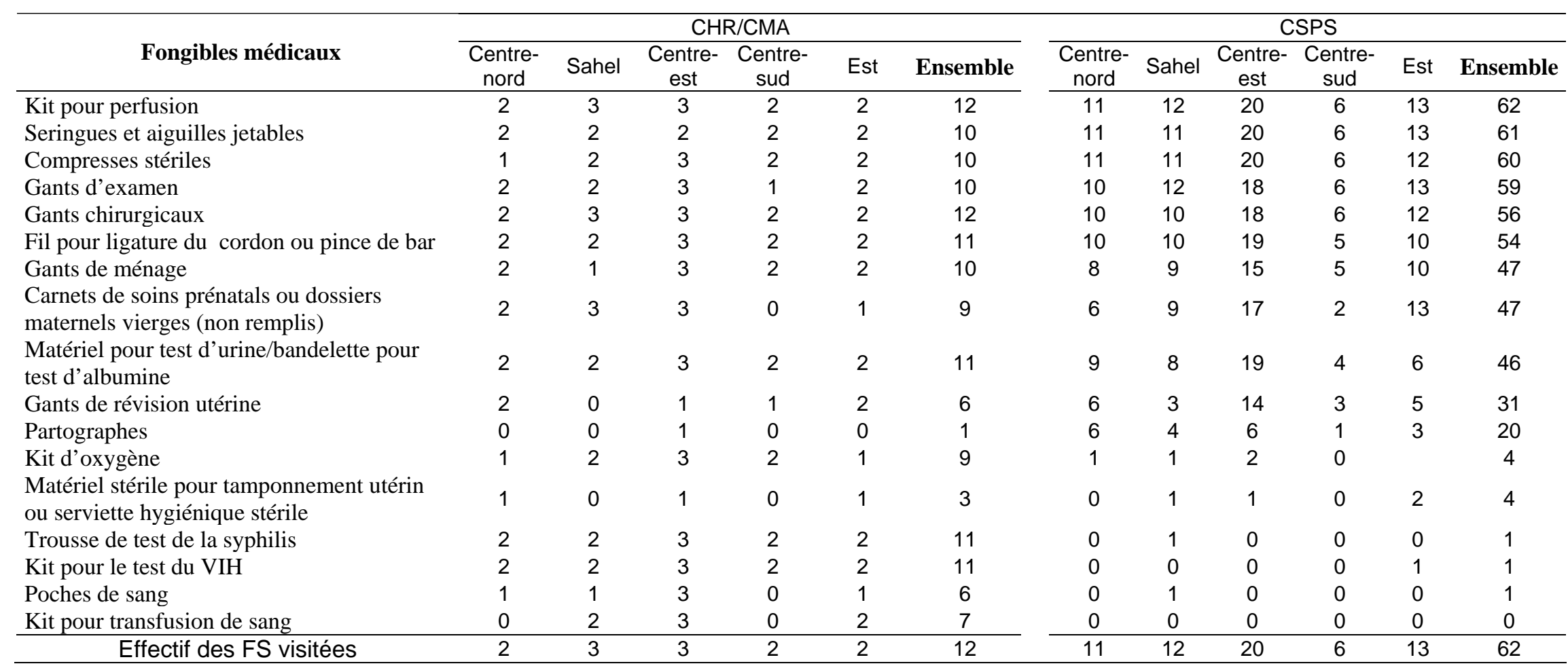




\subsection{Ressources humaines et formation}

Sur les 62 CSPS de la zone d’intervention, plus de la moitié (54,8 \%) répondent aux normes en matière de ressources humaines, c'est-à-dire que la FS dispose d'au moins un infirmier, une accoucheuse auxiliaire et un AIS ou à défaut un manœuvre. Il y a donc lieu de travailler à la normalisation des FS, pour une meilleure prise en charge des patients. Pour 22,6 \% des CSPS, le fonctionnement se fait avec un nombre de prestataires inférieur à 3 agents et il en est autant pour les CSPS avec 3 agents ou plus sans avoir tous les profils d'agents requis pour un CSPS. Dans les régions de l'Est, du Centre-nord et du Centre-sud, moins de la moitié des FS répondent aux normes (Tableau 8) et cette différence est significative.

Tableau 8 : Proportion des CSPS selon la disponibilité du personnel

\begin{tabular}{|c|c|c|c|c|c|c|}
\hline & Centre-nord & Sahel & Centre-est & Centre-sud & Est & Ensemble \\
\hline \multicolumn{7}{|l|}{ Moins de 3 agents } \\
\hline Effectif & 4 & 2 & 3 & 3 & 2 & 14 \\
\hline$\%$ & 36,4 & 16,7 & 15 & 50 & 15,4 & 22,6 \\
\hline \multicolumn{7}{|l|}{3 agents ou plus sans norme } \\
\hline Effectif & 2 & 4 & 2 & 1 & 5 & 14 \\
\hline$\%$ & 18,2 & 33,3 & 10 & 16,7 & 38,5 & 22,6 \\
\hline \multicolumn{7}{|l|}{ Norme et plus } \\
\hline Effectif & 5 & 6 & 15 & 2 & 6 & 34 \\
\hline$\%$ & 45,5 & 50 & 75 & 33,3 & 46,2 & 54,8 \\
\hline Total CSPS & 11 & 12 & 20 & 6 & 13 & 62 \\
\hline \multicolumn{7}{|c|}{ Type de personnel absent dans les FS qui ne sont pas aux normes } \\
\hline Sages-femmes/Maïeuticiens & 100,0 & 75,0 & 100,0 & 100,0 & 50,0 & 84,0 \\
\hline IDE & 16,7 & 16,7 & 20,0 & 25,0 & 0,0 & 14,8 \\
\hline Accoucheuses auxiliaires & 66,7 & 20,0 & 20,0 & 50,0 & 50,0 & 42,3 \\
\hline Infirmiers brevetés & 50,0 & 40,0 & 60,0 & 50,0 & 16,7 & 42,3 \\
\hline Agents itinérants de santé & 33,3 & 83,3 & 80,0 & 50,0 & 83,3 & 66,7 \\
\hline Total CSPS & 6 & 6 & 5 & 4 & 7 & 28 \\
\hline
\end{tabular}

Dans les FS qui n'ont pas la norme en personnel en nombre et en qualité (45,2 \%), il n’y a en général pas de sages-femmes. Cela est constaté dans toutes les FS du Centre-nord, du Centreest et du Centre-sud.

Dans plus du tiers de ces FS qui n'ont pas la norme en personnel en nombre et en qualité, il n'y a pas d'accoucheuses auxiliaires, ni d'infirmiers brevetés. Les AIS sont absents dans 2 de ces FS sur 3 (Tableau 8).

Dans les centres de références, les thèmes les plus couverts à travers les formations reçues en SR par les prestataires au cours des 12 derniers mois précédents l'enquête portent sur l'accouchement, la consultation postnatale, les soins post abortum, l'IEC PF, l'IEC IST autres que VIH/SIDA, les autres soins curatifs aux adultes, l'IEC VIH/SIDA, le traitement des IST autres que VIH/SIDA, la consultation prénatale, la consultation pour infécondité/infertilité, la contraception, l'approvisionnement des méthodes fournies aux clientes et le diagnostic laboratoire des IST autres que VIH.

Les thèmes tels que la PTME, la PCIME, les services PF aux hommes, la surveillance nutritionnelle pondérale, la récupération nutritionnelle et les soins post-partum (suite de couches) ont été très peu couverts (Tableau 9). 
Au cours des 12 derniers mois, les thèmes les plus dispensés dans les FS du premier échelon sont : la consultation postnatale, la consultation prénatale, la contraception, l'accouchement, les soins post-abortum et la vaccination (mère/enfant). Les autres thèmes tels que les services IST/ SIDA aux femmes, la consultation pour incontinence urinaire chez la femme, la PTME, les tests VIH/SIDA, le diagnostic laboratoire des IST autres que le VIH et les soins postpartum (suite de couches) ont été moins abordés au cours des formations récentes (Tableau 9).

Au regard de ces résultats, il s’avère nécessaire de mener des actions pour une mise à niveau du personnel dans les FS pour les thèmes où le constat présente une situation déficiente. Ces thèmes sont entre autres: la PTME, la PCIME, les services de PF, la surveillance nutritionnelle pondérale, la récupération nutritionnelle et les soins post-partum. 


\section{Tableau 9 : Proportion des prestataires qui ont reçu une formation en SR dans les 12 mois ayant précédé l'enquête par région et par thème}

\begin{tabular}{|c|c|c|c|c|c|c|c|c|c|c|c|c|}
\hline \multirow[b]{2}{*}{ Thèmes de formation } & \multicolumn{6}{|c|}{ CHR/CMA } & \multicolumn{6}{|l|}{ CSPS } \\
\hline & $\begin{array}{l}\text { Centre- } \\
\text { nord }\end{array}$ & Sahel & $\begin{array}{l}\text { Centre- } \\
\text { est }\end{array}$ & $\begin{array}{l}\text { Centre- } \\
\text { sud }\end{array}$ & Est & Ensemble & $\begin{array}{l}\text { Centre- } \\
\text { nord }\end{array}$ & Sahel & $\begin{array}{l}\text { Centre- } \\
\text { est }\end{array}$ & $\begin{array}{l}\text { Centre- } \\
\text { sud }\end{array}$ & Est & Ensemble \\
\hline Accouchement & 6 & 7 & 7 & 6 & 7 & 33 & 23 & 26 & 45 & 12 & 24 & 130 \\
\hline Consultation postnatale & 5 & 7 & 7 & 6 & 7 & 32 & 23 & 25 & 46 & 12 & 25 & 131 \\
\hline Soins Post abortum & 6 & 6 & 7 & 6 & 7 & 32 & 23 & 26 & 45 & 12 & 24 & 130 \\
\hline IEC/ PF & 6 & 7 & 6 & 6 & 7 & 32 & 23 & 26 & 44 & 12 & 24 & 129 \\
\hline IEC IST autres que VIH/SIDA & 6 & 7 & 7 & 5 & 7 & 32 & 21 & 22 & 44 & 11 & 23 & 121 \\
\hline Autres soins curatifs -adulte & 6 & 7 & 5 & 5 & 7 & 30 & 23 & 27 & 37 & 11 & 20 & 118 \\
\hline IEC VIH/SIDA & 6 & 6 & 7 & 5 & 6 & 30 & 21 & 21 & 43 & 11 & 23 & 119 \\
\hline Traitement IST autres que VIH SIDA & 6 & 7 & 6 & 6 & 5 & 30 & 20 & 26 & 44 & 11 & 24 & 125 \\
\hline Consultation Prénatale & 6 & 7 & 7 & 6 & 3 & 29 & 23 & 26 & 46 & 12 & 24 & 131 \\
\hline Consultation Infécondité/ Infertilité & 6 & 7 & 6 & 4 & 6 & 29 & 23 & 13 & 30 & 8 & 20 & 94 \\
\hline Contraception & 6 & 7 & 6 & 6 & 3 & 28 & 23 & 26 & 46 & 12 & 24 & 131 \\
\hline L'approvisionnement des méthodes fournies aux clientes & 6 & 7 & 6 & 6 & 3 & 28 & 23 & 27 & 45 & 11 & 23 & 129 \\
\hline Diagnostic laboratoire des IST autres que VIH & 6 & 7 & 5 & 4 & 6 & 28 & 3 & 2 & 3 & 1 & 7 & 16 \\
\hline Consultation pour incontinence urinaire chez la femme & 6 & 6 & 5 & 4 & 6 & 27 & 14 & 5 & 21 & 7 & 17 & 64 \\
\hline Services PF aux adolescents & 6 & 6 & 5 & 5 & 4 & 26 & 20 & 13 & 33 & 10 & 21 & 97 \\
\hline Services IST/SIDA aux adolescents & 6 & 6 & 5 & 4 & 5 & 26 & 4 & 11 & 27 & 9 & 21 & 72 \\
\hline Services IST/ SIDA hommes & 6 & 6 & 6 & 3 & 5 & 26 & 1 & 14 & 24 & 8 & 21 & 68 \\
\hline Services IST/ SIDA femmes & 6 & 6 & 6 & 3 & 5 & 26 & & 14 & 23 & 9 & 21 & 67 \\
\hline IEC Nutrition & 6 & 4 & 6 & 5 & 3 & 24 & 23 & 26 & 45 & 11 & 24 & 129 \\
\hline Autres soins curatifs enfants & 6 & 5 & 3 & 3 & 7 & 24 & 23 & 27 & 35 & 10 & 19 & 114 \\
\hline Tests VIH/SIDA & 6 & 6 & 3 & 3 & 6 & 24 & 3 & 6 & 7 & 1 & 3 & 20 \\
\hline $\begin{array}{l}\text { Troubles de la sexualité masculine (impuissance sexuelle, } \\
\text { troubles de l'éjaculation, troubles de l'érection, ...) }\end{array}$ & 6 & 4 & 4 & 3 & 5 & 22 & 18 & 16 & 20 & 9 & 15 & 78 \\
\hline Vaccination (mère/enfant) & 6 & 4 & 2 & 6 & 3 & 21 & 23 & 26 & 45 & 12 & 24 & 130 \\
\hline LMD (thérapie de réhydratation Orale) & 6 & 4 & 2 & 4 & 5 & 21 & 23 & 27 & 36 & 11 & 24 & 121 \\
\hline Consultation du nourrisson & 6 & 3 & 3 & 4 & 3 & 19 & 23 & 26 & 44 & 10 & 23 & 126 \\
\hline PTME & 6 & 4 & 4 & 2 & 2 & 18 & 8 & 8 & 16 & 4 & 10 & 46 \\
\hline PCIME & 4 & 4 & 2 & 3 & 4 & 17 & 13 & 14 & 26 & 9 & 18 & 80 \\
\hline Services PF aux hommes & 5 & 1 & 5 & 1 & 4 & 16 & 18 & 11 & 28 & 4 & 19 & 80 \\
\hline Surveillance Nutritionnelle Pondérale & 6 & 0 & 2 & 4 & 1 & 13 & 21 & 26 & 43 & 11 & 23 & 124 \\
\hline Récupération Nutritionnelle & 6 & 0 & 2 & 0 & 2 & 10 & 12 & 21 & 21 & 2 & 17 & 73 \\
\hline Soins Post-Partum (suite de couches) & 0 & 0 & 0 & 0 & 0 & 0 & 0 & 0 & 0 & 0 & 0 & 0 \\
\hline Ensemble des prestataires & 6 & 7 & 7 & 6 & 7 & 33 & 23 & 27 & 46 & 12 & 26 & 134 \\
\hline
\end{tabular}




\subsection{Disponibilité et utilisation du matériel IEC pour les activités de santé maternelle}

Le matériel IEC traitant de différents domaines de la santé ${ }^{2}$ se compose de boîtes à images, de dépliants, d'affiches, de cassettes audio, de cassettes-vidéo, pour les activités d'IEC dans les domaines suivants : la PF, le VIH/SIDA, la CPN et la nutrition. Le matériel IEC disponible dans les FS pour les soins de santé maternelle est essentiellement composé d'affiches (Tableau 10). Les autres types de matériels sont peu disponibles. Les brochures sont inexistantes. Aucun matériel d'IEC n'est disponible dans 4 FS (5,4\%). Ce sont des FS du Centre-nord, du Centre-est, du Centre-sud et de l'Est.

Tableau 10 : Disponibilité et thématiques du matériel IEC pour les prestations de soins de santé maternelle par région

\begin{tabular}{|c|c|c|c|c|c|c|}
\hline Types de matériels & $\begin{array}{c}\text { Centre- } \\
\text { nord }\end{array}$ & Sahel & $\begin{array}{c}\text { Centre- } \\
\text { est }\end{array}$ & $\begin{array}{l}\text { Centre- } \\
\text { sud }\end{array}$ & Est & Ensemble \\
\hline disposant au moins d'un matériel & 12 & 15 & 23 & 6 & 14 & 70 \\
\hline Affiches & 11 & 15 & 23 & 5 & 12 & 66 \\
\hline Fiches counselling & 2 & 1 & 19 & 2 & 5 & 29 \\
\hline Dépliants à emporter & 0 & 2 & 16 & 0 & 4 & 22 \\
\hline Matériel audio & 0 & 0 & 3 & 2 & 0 & 5 \\
\hline Autre & 5 & 3 & 0 & 0 & 0 & 8 \\
\hline \multicolumn{7}{|l|}{ Thématiques } \\
\hline La planification familiale & 10 & 14 & 23 & 6 & 14 & 67 \\
\hline Les IST/VIH/SIDA & 8 & 13 & 22 & 6 & 13 & 62 \\
\hline L'allaitement maternel & 6 & 11 & 9 & 2 & 3 & 31 \\
\hline La SRAJ & 1 & 2 & 12 & 2 & 2 & 19 \\
\hline L'excision & 3 & 6 & 2 & & 6 & 17 \\
\hline La nutrition ou l'anémie prénatale & 5 & 2 & 3 & 4 & 1 & 15 \\
\hline $\begin{array}{l}\text { Les signes d'alarme de complication de } \\
\text { l'accouchement }\end{array}$ & 0 & 2 & 9 & 0 & 2 & 13 \\
\hline Les soins du nouveau-né & 0 & 2 & 7 & 0 & 4 & 13 \\
\hline La PTME & 4 & 1 & 4 & 1 & 3 & 13 \\
\hline $\begin{array}{l}\text { Les signes d'alarme de complication de la } \\
\text { grossesse }\end{array}$ & 0 & 2 & 9 & 0 & 1 & 12 \\
\hline Le CDV & 0 & 1 & 6 & 1 & 2 & 10 \\
\hline $\begin{array}{l}\text { Les soins post-avortement (planification } \\
\text { familiale après avortement) }\end{array}$ & 0 & 0 & 3 & 0 & 2 & 5 \\
\hline $\begin{array}{l}\text { Les soins du post-partum (après } \\
\text { l'accouchement) }\end{array}$ & 0 & 0 & 3 & 0 & 0 & 3 \\
\hline Effectif FS & 13 & 15 & 23 & 8 & 15 & 74 \\
\hline
\end{tabular}

Les thèmes abordés dans les supports d'IEC disponibles dans les FS sont en général centrés sur la SR (Tableau 10). La planification familiale et les IST/VIH/SIDA sont les plus présents. Les autres thèmes semblent être négligés car moins présents. Il s’agit surtout des soins du post-partum (après l'accouchement), des soins post-avortement (planification familiale après avortement), du CDV, des signes d'alarme de complications de l'accouchement, etc.

\subsection{Activités d'IEC sur la santé maternelle et les causeries éducatives}

Les clientes venues en consultation ont reçu des conseils. On note que $57 \%$ des clients affirment avoir reçu des conseils lors de la consultation (Graphique 2). Les informations et/ou conseils tels que les règles d'hygiène et de propreté, la nutrition, le régime alimentaire

\footnotetext{
${ }^{2}$ Voir aussi MS : Politique et normes de services de santé de la reproduction, septembre 2000
} 
pendant la grossesse, l'alimentation du nouveau-né et de l'enfant et les bonnes pratiques du sevrage sont les thèmes abordés, mais ils restent dans des proportions très faibles car peu de clients en ont bénéficié (moins de 10 personnes). L'allaitement exclusif au sein n'est pas abordé.

Graphique 2 : Proportion des clientes ayant recu des conseils lors de la consultation par région

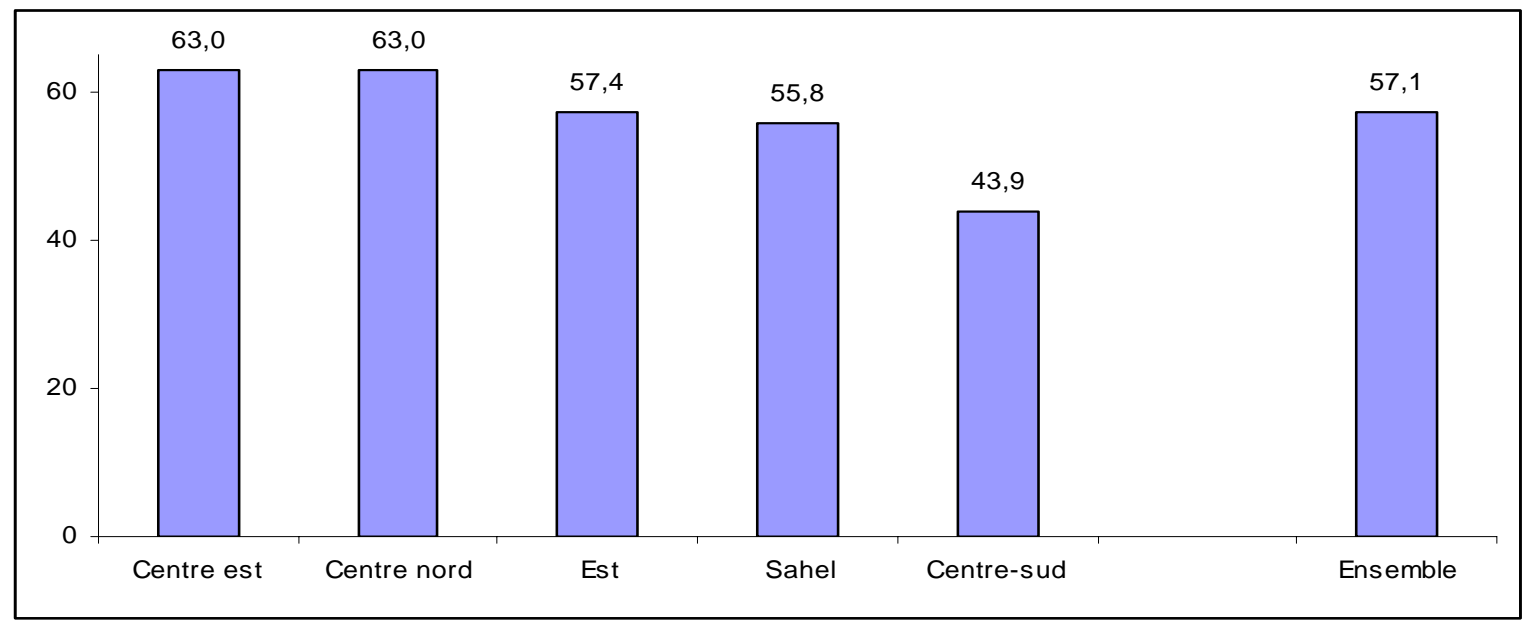

Sur les 245 clients dénombrés, il n’y en a que 43 (17,6 \%) qui ont participé à une causerie éducative organisée le jour de la consultation (graphique 3). Les thèmes abordés lors de ces causeries portaient sur la consultation prénatale (51,2 \%), le VIH/SIDA (37,2 \%) et l'hygiène (23,3 \%). Les thèmes comme la PF, l'accouchement, les IST autres que VIH/SIDA, la vaccination, la surveillance nutritionnelle et pondérale, l'infécondité et l'infertilité, l'allaitement maternel, les bonnes pratiques du sevrage, la nutrition n'ont pas été abordés. Le postnatal, la LMD, les soins post-abortum, les services curatifs ont été très peu abordés (seulement 2 clients).

Graphique 3 : Proportion des clientes ayant participé à des causeries éducatives le jour de la consultation par région

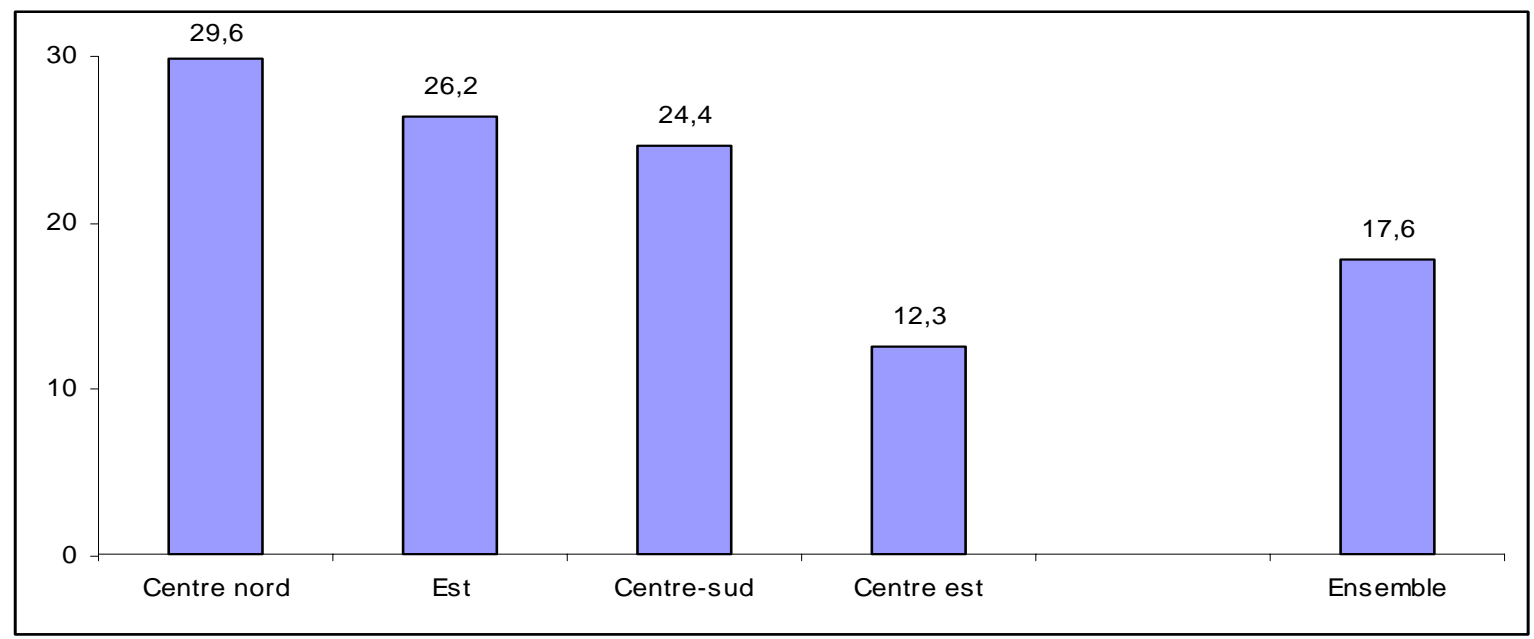

La non disponibilité du matériel IEC justifierait son non usage. On relève que très peu de femmes affirment en avoir reçu. L’utilisation du matériel IEC par les Prestataires est faible. 
Ce qui concorde avec le fait que le matériel IEC n'est pas offert aux femmes venues en consultation.

\subsection{Gestion et supervision de la FS}

L'appréciation des capacités de gestion et de la supervision des services a porté sur la disponibilité des produits de SR à travers l'enregistrement de rupture de stock, l'effectivité de la supervision des FS et la disponibilité des documents de référence pour la planification. Ce sont des éléments de la qualité de la gestion dans les FS.

\section{Disponibilité des produits de SR}

La rupture de stock de médicaments est une information de la disponibilité des produits de SR. Elle a été effective dans 3 FS sur 5 au cours des 12 derniers mois (Graphique 4). Elle concerne plus les FS du Centre-nord.

Graphique 4 : Proportions des FS ayant connu une rupture de stock de médicaments et consommables de SR au cours des 12 derniers mois par région

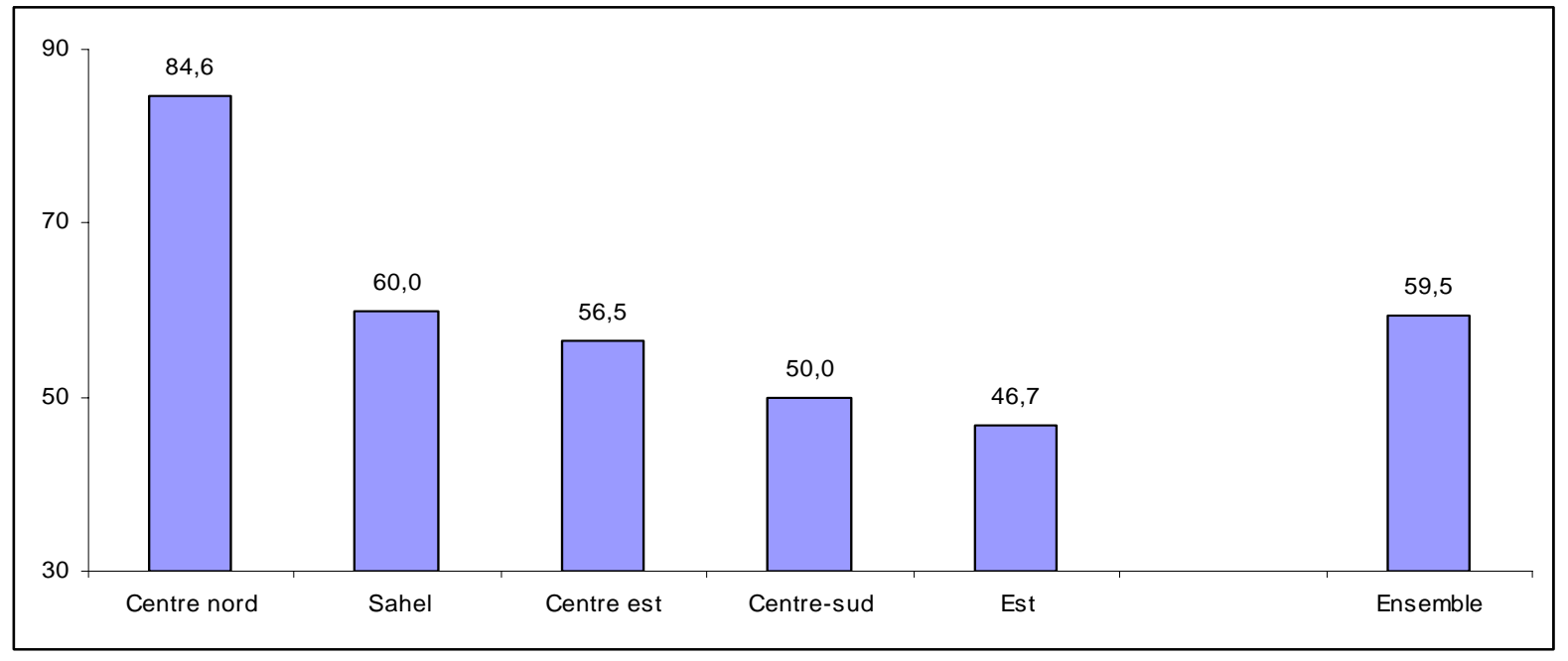

De nombreux produits sont concernés par ces ruptures de stock. Il s'agit de : kit IST, injectables, pilule, condoms, kit césarienne et gants d'examen, pour ce qui est relatif à la SR. D'autres produits tels que la SRO, la quinine $300 \mathrm{mg}$ cp, le Fer + AF $225 \mathrm{mg}$ cp, le Cotrimoxazole 480mg cp, l’AAS 500mg cp, le Paracétamol, la Chloroquine 100 mg cp, le Métronidazole $250 \mathrm{mg}$ cp et le Mébendazole ont aussi connu des ruptures de stock.

Le réapprovisionnement se fait dans la majorité des FS à intervalles réguliers (52,7\%), ou quand le stock atteint le seuil de sécurité (43,2 \%). D’une manière générale, les contraceptifs oraux $(1,4 \%)$ et les injectables $(5,4 \%)$ sont très peu disponibles dans la majorité des FS. Les autres types de contraceptifs sont plus disponibles : spermicides $(100,0 \%)$, collier $(87,8 \%)$, DIU (77,0 \%) et condom féminin (74,3 \%).

\section{Supervision}

L'amélioration de la performance des agents de santé sur le terrain, la motivation et le soutien des prestataires, et l'amélioration des conditions de travail passent par la supervision du personnel. Suivant les normes de périodicité de supervision, la majorité (62,2\%) des FS ont reçu une supervision au cours des 3 derniers mois. Les prestataires qui ont effectué des 
supervisions sont dans des proportions plus faibles (56,4\%), avec cependant des variations d’une région à l'autre (Graphique 5).

\section{Graphique 5 : Proportions de prestataires effectuant des supervisions par région}

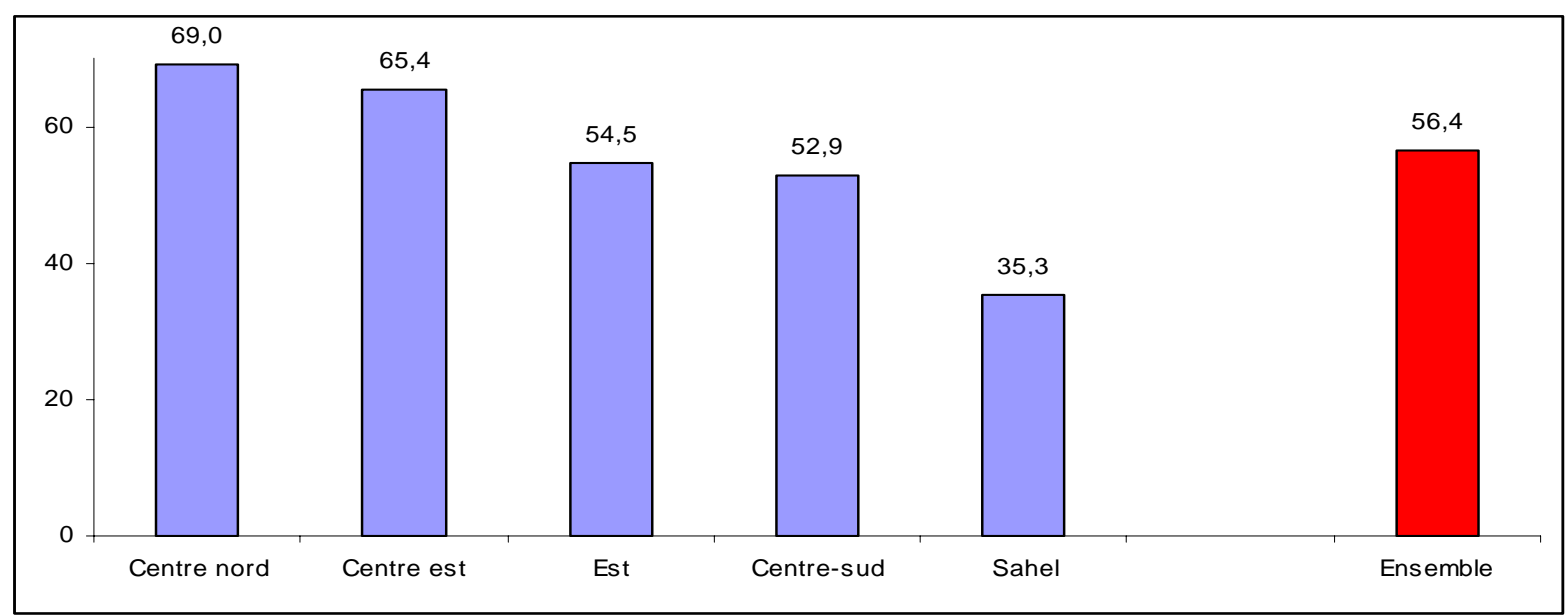

L'utilisation d'outils standardisés pour la supervision n'est pas de règle (Graphique 6). Moins d'un prestataire sur 5 dispose d'une grille de supervision.

\section{Graphique 6 : Proportions de prestataires disposant de grille de supervisions par région}

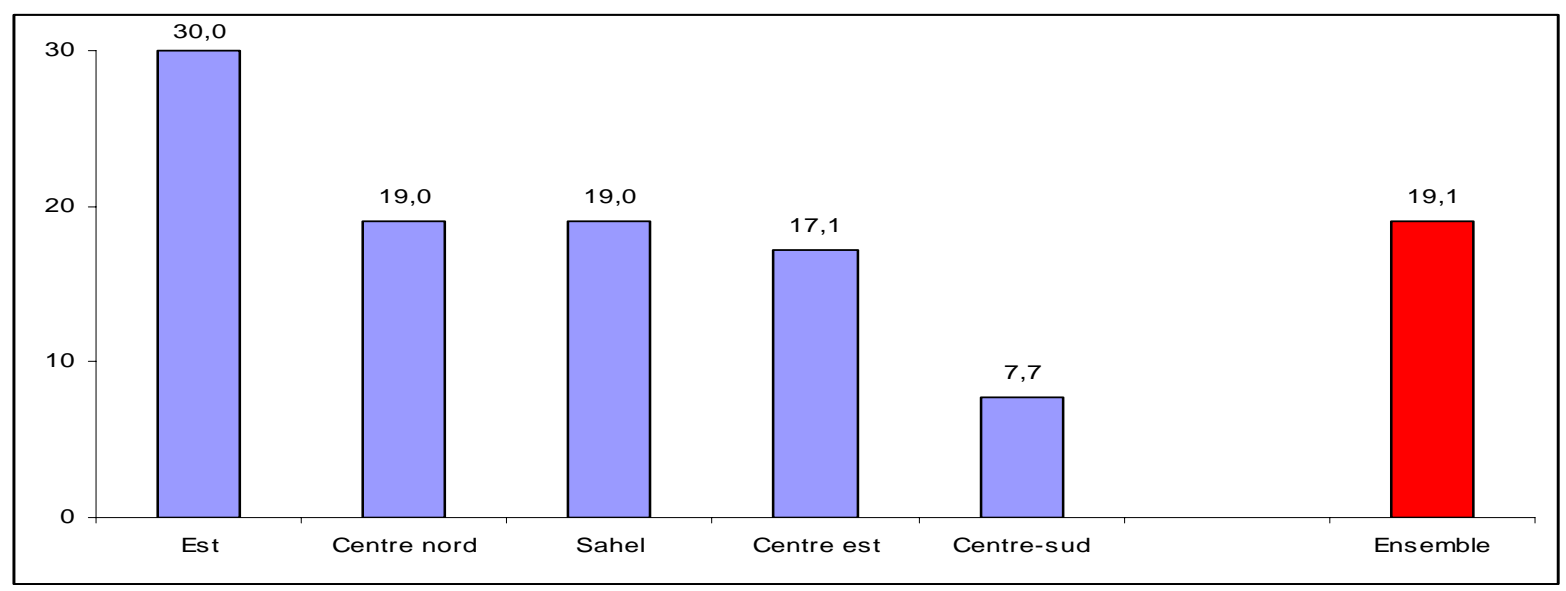

\section{Disponibilité des documents de référence}

Le plan d'action et le plan de développement sanitaire sont partout disponibles. A l'inverse, bon nombre de documents sont peu disponibles dans les FS. Pour 18 des FS (22,3 \%), aucun document n'est disponible. Ce qui revient à dire que dans 1 FS sur 5, il n'y a pas de document de référence. Le plan stratégique de santé des jeunes est un document qui est très peu disponible (5 FS). Il est cependant très utile pour une meilleure prise en compte des préoccupations des adolescents.

Les documents tels que les protocoles cliniques (guides de diagnostique et traitement, algorithmes/ordinogrammes) sont disponibles dans la plupart des FS (67/74). La faible disponibilité des documents de référence s'accompagne de leur faible utilisation par les prestataires (40,4 \%). Dans 47,3 \% des FS, les prestataires les consultent en cas de besoin et pour $40,5 \%$ c'est de façon systématique. 


\section{Existence des COGES fonctionnels}

Dans toutes les FS il n'existe pas un COGES fonctionnel. En général, dans la zone d'intervention, les COGES sont fonctionnels dans environ 9 FS sur 10. Leur absence est plus marquée dans les régions de l'Est et du Centre-sud (Graphique 7). L'existence des COGES dans les FS est une nécessité pour une meilleure gestion dans celles-ci. Il y a lieu que les COGES fonctionnels soient créés dans les FS qui n’en disposent pas.

\section{Graphique 7 : Existence de COGES fonctionnel par région}

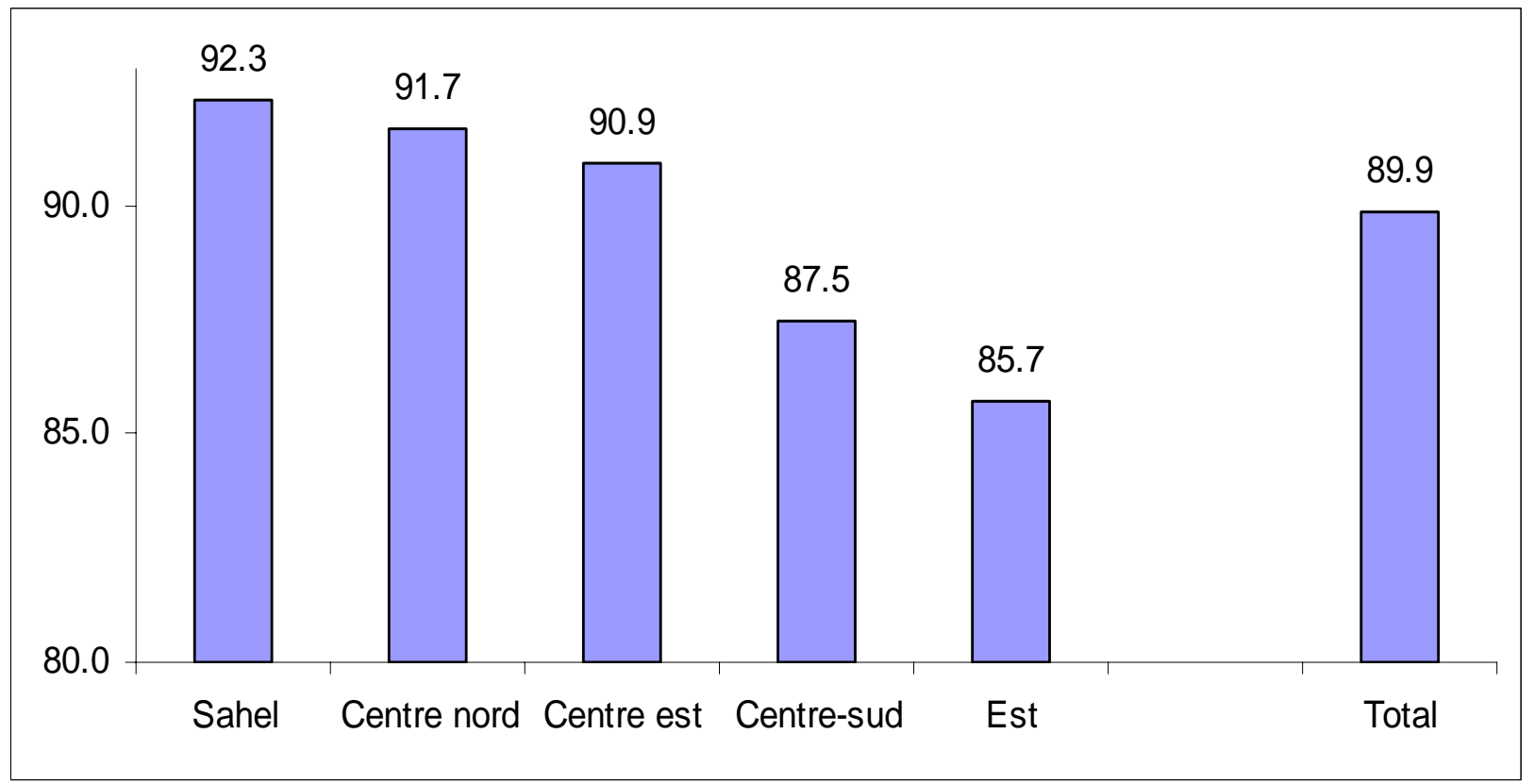




\section{QUALITE DES SERVICES SR DANS LES FS}

\subsection{Accessibilité des services}

L’appréciation de l'accessibilité des services dans les FS s'est intéressée aux heures d'ouverture des FS et aux coûts. En général, les clients ont été satisfaits des heures d’ouverture (Graphique 8. Les différences entre les régions ne sont pas significatives.

\section{Graphique 8 : Proportions des clients satisfaits des heures d'ouverture des FS par région}

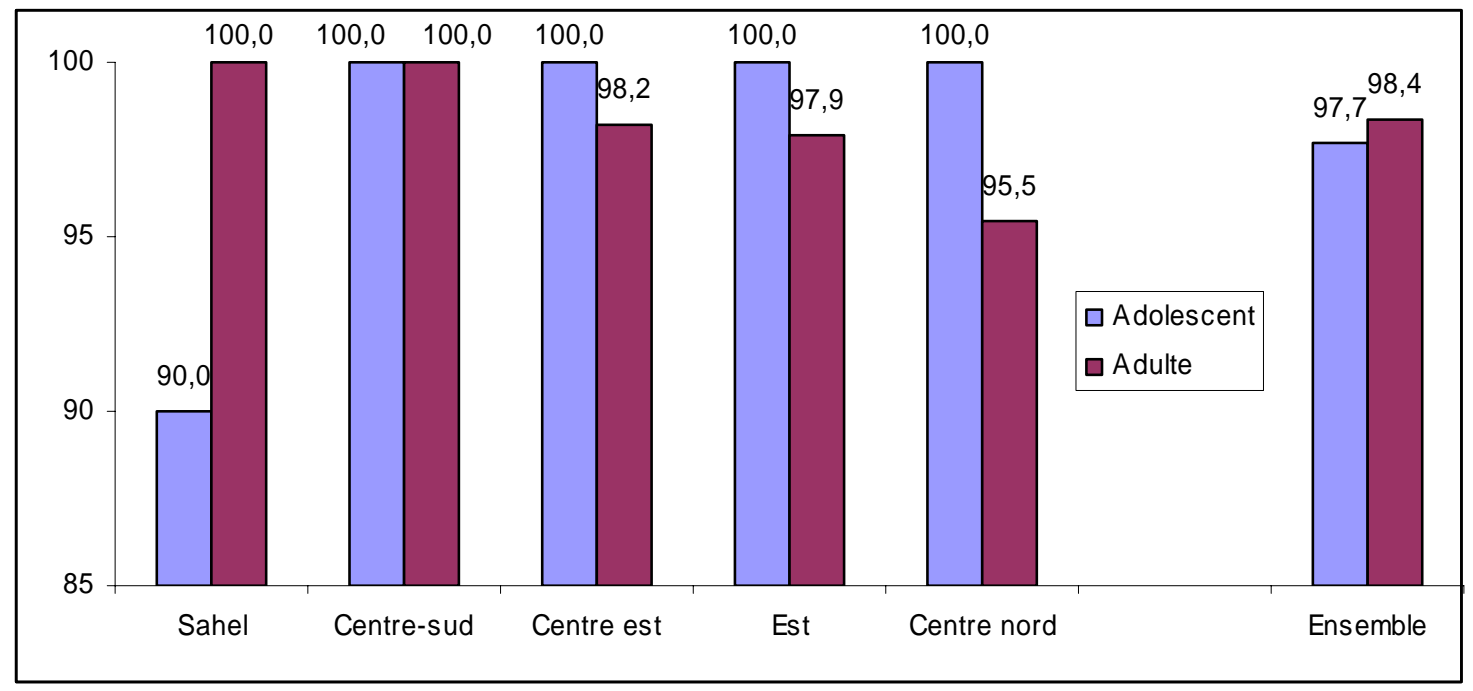

S’agissant des coûts, il apparaît que le carnet de santé et le carnet de CPN ne sont pas partout obtenus gratuitement (Graphique 9. La gratuité est même très variable dans une région et d'une région à l'autre. Les différences entre les régions ne sont cependant pas significatives.

\section{Graphique 9: Proportions des clients ayant reçu gratuitement le ticket de consultation} pour la CPN par région

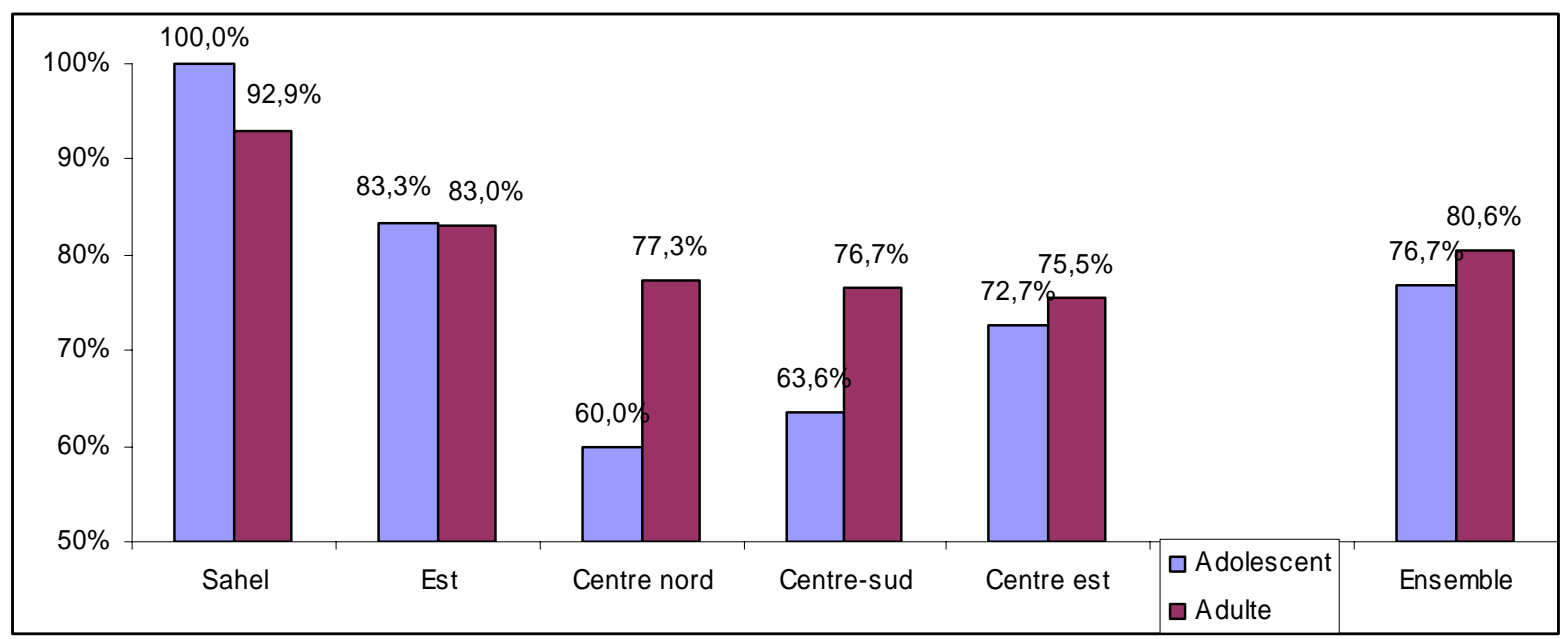

On constate qu'au Sahel, les adolescents sont peu satisfaits des heures d'ouverture des FS (90 $\%)$ comparativement aux autres régions. 


\subsection{Performances techniques des prestataires}

Les performances techniques des prestataires sont évaluées à partir de l'interrogatoire, de l'examen physique, de la prévention des infections et des tests et examens para cliniques. Ces performances sont appréciées au cours des interactions lors des consultations en CPN, en PF, en IST et en LMD.

\section{- Consultation prénatale}

En consultation prénatale, il ressort qu'au cours de l'interrogatoire d'une cliente les prestataires s’intéressent essentiellement aux plaintes de la cliente (80,6 \%), au motif de la visite (58,3 \%) et à l’administration du vaccin contre le tétanos (53,7 \%).

Pour moins de $1 / 4$ des prestataires, les éléments suivants sont plus ou moins négligés : les antécédents médicaux et chirurgicaux de la cliente, la date du dernier accouchement, l'existence de pathologies (Diabète, HTA, Cardiopathie, etc.), l'existence d'un accouchement dystocique antérieur, l'issue de la dernière grossesse et des renseignements sur les allaitements passés (Tableau 11).

Par rapport aux adolescents, on remarque que seulement 1/3 des prestataires s'intéressent à l'âge des clients, élément pertinent pour considérer les préoccupations de cette catégorie de population. Ce qui amènerait à conclure que les adolescents sont traités comme des adultes par beaucoup de prestataires. Il faut souligner que pour nombre de prestataires, les âges des anciens clients étant inscrits dans leurs carnets de santé, il n'était plus opportun de les interroger sur le sujet.

Au cours de l'examen physique, les gestes essentiels sont exécutés. Cependant, d'autres ne le sont pas. L'explication de la procédure d'examen avant celui-ci et l'explication des résultats à la cliente sont exécutées par moins du 1/44 des prestataires (Tableau 11).

\section{- Consultation de planification familiale}

Au cours de l'interrogatoire en consultation pour PF, les prestataires s'intéressent surtout au motif de la visite $(96,6 \%)$, la date des dernières règles $(86,2 \%)$ et l'âge de la cliente $(69,0$ \%). Il apparaît que pour 3 prestataires sur 10, l'âge des clients n'est pas pris en compte, ce qui ne permet pas de faire la distinction des adultes et des adolescents pour un councelling différencié et pour une offre de service approprié.

Les éléments suivants sont pris en compte lors de l'examen physique : la prise de la tension artérielle, la pesée de la cliente, la recherche d'anémie, l'examen pelvien, la recherche de signes d'IST, d'œdèmes des membres et de varices. Par contre, certaines bonnes pratiques de prévention de l'infection (lavage des mains et décontamination du matériel et des gants), l'information sur la procédure d'examen et la communication des résultats ne sont pas respectées (Tableau 11). 
Tableau 11 : Proportions de prestataires selon les éléments d'investigation de la consultation en CPN et en PF

\begin{tabular}{|c|c|c|c|}
\hline \multicolumn{4}{|c|}{ Consultation prénatale } \\
\hline \multicolumn{2}{|l|}{ Interrogatoire } & \multicolumn{2}{|l|}{ Examen physique } \\
\hline Si la cliente a des plaintes & 80,6 & Pesé la cliente & 98,3 \\
\hline Si la cliente a des plaintes & 80,6 & Pesé la cliente & 98,3 \\
\hline Le motif de la visite & 58,3 & Mesuré la hauteur utérine & 97,7 \\
\hline Si elle a reçu son vaccin contre le tétanos & 53,7 & Palpé l’abdomen & 96,0 \\
\hline Le nombre de grossesses antérieures & 35,4 & Procédé à un toucher vaginal & 96,0 \\
\hline Le nombre d'enfants vivants et bien portants & 35,4 & Examiné les conjonctives & 95,4 \\
\hline L’âge de la cliente? & 32,6 & $\begin{array}{l}\text { Ausculté l'abdomen pour détecter les bruits du cœur } \\
\text { fœtal }\end{array}$ & 93,7 \\
\hline Le nombre d'avortements & 31,4 & Pris la tension artérielle & 88,0 \\
\hline Si elle a déjà consulté pour cette grossesse & 30,3 & Examiné les jambes (œdèmes, varices) & 84,6 \\
\hline Le nombre de mort-nés & 25,7 & Recherché des signes d'IST & 63,4 \\
\hline Si elle ressent les mouvements actifs de son bébé & 25,1 & Fait un examen mammaire & 61,7 \\
\hline Le statut matrimonial de la cliente? & 23,4 & Inspecter les parties génitales & 41,1 \\
\hline La date de ses dernières règles & 18,3 & Pris la taille de la cliente & 38,3 \\
\hline Prise d’autres médicaments actuellement & 12,6 & Demandé à la femme d’aller uriner & 34,9 \\
\hline Les antécédents médicaux et chirurgicaux de la cliente? & 8,6 & $\begin{array}{l}\text { Informé la cliente des résultats de l'examen } \\
\text { physique }\end{array}$ & 29,7 \\
\hline La date du dernier accouchement & 6,9 & $\begin{array}{l}\text { Informé du déroulement de l'examen avant de } \\
\text { procéder à l'examen }\end{array}$ & 27,4 \\
\hline L’existence de pathologie telle que (Diabète, HTA, Cardiopathie etc.) & 4,0 & Pris le pouls de la cliente & 5,7 \\
\hline L'existence d'un accouchement antérieur dystocique & 3,4 & Référé s'il s’agit d’une grossesse à risque & 1,1 \\
\hline L'issue de la dernière grossesse & 3,4 & Fait l'échographie (CPN1, CPN3) & 1,1 \\
\hline Des renseignements sur les allaitements passés & 1,7 & Utilisé le spéculum & 0,6 \\
\hline Effectif total prestataires & 175 & Effectif total prestataires & 175 \\
\hline \multicolumn{4}{|c|}{ Consultation de PF } \\
\hline Le motif de la visite & 96,6 & Pris de la tension artérielle & 82,8 \\
\hline Dates des dernières règles & 86,2 & Pesé la cliente & 75,9 \\
\hline L’âge de la cliente & 69,0 & Porté des gants selon la technique indiquée & 75,9 \\
\hline Antécédents gynéco-obstréticaux cliente & 58,6 & Fait un examen pelvien & 69,0 \\
\hline L’âge du dernier enfant & 58,6 & Recherché des signes IST & 69,0 \\
\hline Les caractéristiques du cycle menstruel (régulier ou irrégulier) & 55,2 & Fait un toucher vaginal combiné au palpé? & 69,0 \\
\hline L'allaitement au sein & 42,9 & Vérifié si la cliente est anémiée & 65,5 \\
\hline Le désir d'autres enfants & 37,9 & Recherché des œdèmes, des varices & 58,6 \\
\hline La présence de saignements ou de saignements en dehors des règles & 37,9 & Inspecté les zones génitales externes? & 58,6 \\
\hline Inquiétudes cliente sur méthodes de PF ? & 24,1 & Fait un examen mammaire & 44,8 \\
\hline Discussion de PF client et son partenaire & 24,1 & Lavé ses mains après l'examen? & 44,8 \\
\hline La présence d’essoufflement ou dyspnée & 20,7 & Fait un examen au spéculum ? & 37,9 \\
\hline La présence des douleurs au bas ventre & 20,7 & $\begin{array}{l}\text { Décontaminé le matériel et les gants après } \\
\text { l'examen? }\end{array}$ & 34,5 \\
\hline La présence des migraines & 14,3 & Lavé ses mains avant l'examen? & 31,0 \\
\hline la consommation de cigarette/tabac & 13,8 & Après l'examen, informé la cliente des résultats ? & 28,6 \\
\hline La présence des leucorrhées inhabituelles & 13,8 & Porté des doigtiers? & 27,6 \\
\hline Présence démangeaisons vulvo vaginales & 10,3 & Fait aucune de ces actions & 24,1 \\
\hline La présence de drépanocytose & 6,9 & Informé la cliente de la procédure de l’examen ? & 20,7 \\
\hline Les inquiétudes de la cliente concernant les IST ou le SIDA & 6,9 & Fait ou référé pour un test de grossesse & 6,9 \\
\hline Prise des médicaments contre l'épilepsie & 0,0 & Effectif total prestataires & 29 \\
\hline Prise médicaments contre la tuberculose & 0,0 & & \\
\hline Effectif total prestataires & 29 & & \\
\hline
\end{tabular}




\section{- Consultation en IST}

Les prestataires interrogent les clients sur le motif de la visite $(96,4 \%)$ et la présence de douleurs pelviennes (75,0 \%). L'existence d'un comportement sexuel à risque, la présence de tuméfaction du scrotum et la présence de dyspareunie sont les aspects les plus négligés (Tableau 12). La présence de bubon inguinale n’est pas du tout investiguée.

En consultation IST, l'examen des zones génitales externes et le port de gants sont essentiels. Certaines bonnes pratiques de prévention de l'infection (lavage des mains, décontamination du matériel) et l'information du client sur la procédure de l'examen ne sont pas observées par les prestataires (Tableau 12).

\section{Tableau 12 : Proportions de prestataires selon les éléments d'investigation de la consultation en IST et en LMD}

\begin{tabular}{|c|c|c|c|}
\hline \multicolumn{4}{|c|}{ Consultation IST } \\
\hline \multicolumn{2}{|l|}{ Interrogatoire } & \multicolumn{2}{|l|}{ Examen physique } \\
\hline Le motif de la visite & 96,4 & Examiné les zones génitales externes & 85,7 \\
\hline Présence des douleurs pelviennes & 75,0 & Porté des gants? & 82,1 \\
\hline La situation matrimoniale du (de la) client (e) ? & 64,3 & Fait un toucher vaginal ? & 82,1 \\
\hline Présence d'écoulement vaginal/urétral & 64,3 & Fait un examen général? & 64,3 \\
\hline L’âge du (de la) client (e)? & 60,7 & Après l'examen, a informé la cliente des résultats & 46,4 \\
\hline Les antécédents médicaux (IST) du client (e) ? & 42,9 & S’est lavé les mains après examen & 46,4 \\
\hline Présence de douleur au cours de la miction & 42,9 & Informé la cliente de la procédure de l’examen? & 42,9 \\
\hline Le nombre de partenaires du (de la) client (e) ? & 28,6 & Fait un examen au spéculum? & 28,6 \\
\hline L'existence d'un comportement sexuel à risque & 17,9 & S'est lavé les mains avant l'examen? & 25,0 \\
\hline Présence de tuméfaction du scrotum & 7,1 & Décontaminé le matériel & 25,0 \\
\hline Présence de dyspareunie & 3,6 & Porté des doigtiers? & 21,4 \\
\hline Présence de bubon inguinale & 0,0 & Aucune de ces actions & 7,1 \\
\hline Effectif total prestataires & 28 & Effectif total prestataires & 28 \\
\hline \multicolumn{4}{|c|}{ Consultation de diarrhée } \\
\hline S’il y a des signes associés (toux, vomissements, fièvre) & 100,0 & Pris la température & 100,0 \\
\hline Le motif de la visite & 97,1 & Examiné les muqueuses & 88,6 \\
\hline Quel était l'aspect des selles? & 97,1 & Examiné les yeux & 85,7 \\
\hline La date de début de la diarrhée & 94,3 & Recherché un pli de déshydratation & 80,0 \\
\hline Le nombre de selles quotidiennes & 91,4 & $\begin{array}{l}\text { Apprécié l'état général de l'enfant (apathie, } \\
\text { irritabilité) }\end{array}$ & 77,1 \\
\hline L'âge de l'enfant & 88,6 & Essayé de calmer l'enfant durant l'examen & 60,0 \\
\hline S’il y a présence du sang dans les selles & 77,1 & Pesé l’enfant & 42,9 \\
\hline Si l'enfant fait une diarrhée présentement & 74,3 & Examiné de la paume des mains & 42,9 \\
\hline Le statut vaccinal de l'enfant & 57,1 & Examiné de la plante des pieds & 37,1 \\
\hline Les traitements déjà entrepris & 54,3 & Recherché une dépression de la fontanelle & 22,9 \\
\hline A-t-il exploré les causes probables & 54,3 & Donné à boire à l'enfant (\%) & \\
\hline Si l'enfant tête et mange normalement & 45,7 & Effectif total prestataires & 35 \\
\hline Si l’enfant est allaité au sein & 42,9 & & \\
\hline Si l'enfant boit normalement & 37,1 & & \\
\hline Questions sur les antécédents médicaux et chirurgicaux de l’enfant & 28,6 & & \\
\hline Si l'enfant est sevré & 17,1 & & \\
\hline Effectif total prestataires & 35 & & \\
\hline
\end{tabular}


- Consultation de diarrhée

Les prestataires s'intéressent surtout au motif de la visite (97,1 \%), à l'aspect des selles (97,1 \%), aux signes associés (100\%), à la date de début de la diarrhée (94,3\%) et à l'âge de l'enfant (88,6 \%) lors de l'interrogatoire en consultations pour diarrhées infantiles (Tableau 11).

L’appréciation de l'état général de l'enfant, la prise de la température, la recherche d'un pli cutané et l'examen des muqueuses sont souvent exécutés durant l'examen physique lors de la consultation de diarrhée. Par contre, la pesée de l'enfant et le conseil de donner à boire à l'enfant ne sont pas appliqués (Tableau 12).

Les résultats de l'observation laissent apparaître certaines lacunes dans les procédures de l'interrogatoire et de l'examen physique dans les différents types de services. Les corrections de ces lacunes requièrent une supervision régulière et un recyclage ou une mise à niveau des prestataires.

\section{- Prévention des infections}

S'agissant de la prévention des infections, il est apparu que les règles de la prévention des infections (PI) ne sont pas toujours et partout observées. En consultation de PF et d'IST, il y a moins de la moitié des prestataires qui se lavent les mains après l'examen. Cela se comprend dans la mesure où la majorité a porté des gants. De plus les différentes FS ne disposent pas d'une source d'approvisionnement en eau, ce qui limite certaines pratiques.

\section{- Tests et examens para cliniques}

La recherche d'albumine/sucre dans les urines est l'examen para clinique le plus demandé ou réalisé par les prestataires au cours de la CPN. Les examens qui relèvent des FS équipées d'un laboratoire sont peu ou pas prescrits par les prestataires (Tableau 13). Le taux d'hémoglobine, le test d'EMMEL, l'électrophorèse, le KOP et l'échographie lors de la précédente CPN n’apparaissent pas comme test ou examen prescrit par les prestataires.

Tableau 13 : Proportions des prestataires observés ayant demandé ou réalisé des tests ou examens paracliniques pendant la consultation de CPN par type de formation sanitaire

\begin{tabular}{lccc}
\hline \multicolumn{1}{c}{ Tests ou examens paracliniques } & $\begin{array}{c}\text { Centre Hospitalier } \\
\text { Régional (CHR) }\end{array}$ & $\begin{array}{c}\text { Centre Médical avec Antenne } \\
\text { chirurgicale (CMA) }\end{array}$ & $\begin{array}{c}\text { Centre de Santé et de } \\
\text { Promotion Sociale (CSPS) }\end{array}$ \\
\hline Groupage SANGUIN $\backslash$ RH & 25,0 & 15,8 & 6,6 \\
Recherche d'albumine/sucre urines & 25,0 & 73,7 & 60,5 \\
BW (TPHA, RPR) & 25,0 & 26,3 & 7,9 \\
Serologie VIH & - & - & 1,3 \\
Informé la cliente de ses résultats de & - & 15,8 & 5,3 \\
laboratoire & 12 & 21 & 134 \\
\hline Effectif de FS & & & 5,3 \\
\hline
\end{tabular}

\subsection{Expérience et formation en matière de SR}

La contraception, l'IEC PF, les services PF aux adolescents, la consultation prénatale, l'accouchement, les soins post-partum (suite de couches), les soins post abortum, la consultation postnatale, la vaccination (mère/enfant), la surveillance nutritionnelle et pondérale, l'IEC nutrition, la LMD (thérapie de réhydratation orale), l'IEC VIH/SIDA, l'IEC IST autres que VIH/SIDA, le traitement des IST autres que le VIH/SIDA, les autres soins curatifs adultes et enfants, l'approvisionnement des méthodes fournies aux clientes, la consultation du nourrisson sont les services offerts par la majorité des prestataires (Tableau 
14). Les services du paquet minimum d'activités de SR ne sont pas tous offerts. Moins de $50 \%$ des prestataires offrent les services tels que le test du VIH/SIDA, la PTME et le diagnostic de laboratoire des IST autres que le VIH/SIDA (Tableau 14). Les différences observées entre les régions sont significatives.

Tableau 14 : Proportions des prestataires par service fournis aux clients et par région sanitaire

\begin{tabular}{|c|c|c|c|c|c|c|}
\hline Services fournis & $\begin{array}{c}\text { Centre- } \\
\text { nord }\end{array}$ & Sahel & $\begin{array}{l}\text { Centre- } \\
\text { est }\end{array}$ & $\begin{array}{l}\text { Centre- } \\
\text { sud }\end{array}$ & Est & Ensemble \\
\hline Accouchement & 100,0 & 97,1 & 98,1 & 100,0 & 93,9 & 97,6 \\
\hline Soins Post-Partum (suite de couches) & 100,0 & 97,1 & 98,1 & 100,0 & 93,9 & 97,6 \\
\hline Soins Post abortum & 100,0 & 94,1 & 98,1 & 100,0 & 96,9 & 97,6 \\
\hline Consultation postnatale & 96,6 & 94,1 & 100,0 & 100,0 & 97,0 & 97,6 \\
\hline IEC/ PF & 100,0 & 97,1 & 94,3 & 100,0 & 93,9 & 96,4 \\
\hline Consultation Prénatale & 100,0 & 97,1 & 100,0 & 100,0 & 81,8 & 95,8 \\
\hline Contraception & 100,0 & 97,1 & 98,1 & 100,0 & 81,8 & 95,2 \\
\hline L'approvisionnement des méthodes fournies aux clientes & 100,0 & 100,0 & 96,2 & 94,4 & 78,8 & 94,0 \\
\hline Traitement IST autres que VIH SIDA & 89,7 & 97,1 & 94,3 & 94,4 & 87,9 & 92,8 \\
\hline IEC Nutrition & 100,0 & 88,2 & 96,2 & 88,9 & 81,8 & 91,6 \\
\hline IEC IST autres que VIH/SIDA & 93,1 & 85,3 & 96,2 & 88,9 & 90,9 & 91,6 \\
\hline Vaccination (mère/enfant) & 100,0 & 88,2 & 88,7 & 100,0 & 81,8 & 90,4 \\
\hline IEC VIH/SIDA & 93,1 & 79,4 & 94,3 & 88,9 & 87,9 & 89,2 \\
\hline Autres soins curatifs -adulte & 100,0 & 100,0 & 79,2 & 88,9 & 81,8 & 88,6 \\
\hline Consultation du nourrisson & 100,0 & 85,3 & 88,7 & 77,8 & 78,8 & 86,8 \\
\hline LMD (thérapie de réhydratation Orale) & 100,0 & 91,2 & 71,7 & 83,3 & 87,9 & 85,0 \\
\hline Autres soins curatifs enfants & 100,0 & 94,1 & 71,7 & 72,2 & 78,8 & 82,6 \\
\hline Surveillance Nutritionnelle Pondérale & 93,1 & 76,5 & 84,9 & 83,3 & 72,7 & 82,0 \\
\hline Services PF aux adolescents & 89,7 & 55,9 & 71,7 & 83,3 & 75,8 & 73,7 \\
\hline Consultation Infécondité/ Infertilité & 100,0 & 58,8 & 67,9 & 66,7 & 78,8 & 73,7 \\
\hline $\begin{array}{l}\text { Troubles de la sexualité masculine (impuissance sexuelle, troubles } \\
\text { de l'éjaculation, troubles de l'érection, ... }\end{array}$ & 82,8 & 58,8 & 45,3 & 66,7 & 60,6 & 59,9 \\
\hline Services IST/SIDA aux adolescents & 34,5 & 50,0 & 60,4 & 72,2 & 78,8 & 58,7 \\
\hline PCIME & 58,6 & 52,9 & 52,8 & 66,7 & 66,7 & 58,1 \\
\hline Services PF aux hommes & 79,3 & 35,3 & 62,3 & 27,8 & 69,7 & 57,5 \\
\hline Services IST/ SIDA hommes & 24,1 & 58,8 & 56,6 & 61,1 & 78,8 & 56,3 \\
\hline Services IST/ SIDA femmes & 20,7 & 58,8 & 54,7 & 66,7 & 78,8 & 55,7 \\
\hline Consultation pour incontinence urinaire chez la femme & 69,0 & 32,4 & 49,1 & 61,1 & 69,7 & 54,5 \\
\hline Récupération Nutritionnelle & 62,1 & 61,8 & 43,4 & 11,1 & 57,6 & 49,7 \\
\hline PTME & 48,3 & 35,3 & 37,7 & 33,3 & 36,4 & 38,3 \\
\hline Tests VIH/SIDA & 31,0 & 35,3 & 18,9 & 22,2 & 27,3 & 26,3 \\
\hline Diagnostic de laboratoire des IST autres que VIH/SIDA & 31,0 & 26,5 & 15,1 & 27,8 & 39,4 & 26,3 \\
\hline Effectif total prestataires & 29 & 34 & 53 & 18 & 33 & 167 \\
\hline
\end{tabular}

Formation de base

Au cours de leur formation de base, plus de $80 \%$ des prestataires ont reçu des compétences sur la surveillance de la grossesse, l'accouchement et les soins du post-partum, la prévention et la prise en charge de la malnutrition, le PEV (vaccination des enfants et VAT), l'allaitement maternel et la bonne pratique du sevrage, la surveillance nutritionnelle staturo-pondérale, la LMD, la prise en charge des cas d'avortement, les techniques d'IEC, la prévention des infections, le counselling/IEC IST/VIH/SIDA, la formation clinique en IST/VIH/SIDA et les 
soins obstétricaux essentiels (SOE) (Tableau 14). La SRAJ, la gestion de logistique contraceptive, la consultation prénuptiale et préconceptionnelle, la PCIME, la technique de Counselling pour le test de dépistage, la CPN recentrée et la PTME ont été moins abordées au cours des formations de base car peu de prestataires les ont reçues. On observera que les compétences qui font plus défaut s'inscrivent dans des programmes et concepts qui étaient nouveaux (CPN recentrée, PCIME et PTME). Il ne s'est donc pas encore passé suffisamment de temps pour que les prestataires s'en approprient. On remarquera que plus du tiers des prestataires n'ont pas été formés en soins obstétricaux et néonataux d'urgence (SONU). Un peu plus de 1/3 des prestataires y ont été recyclés.

\section{Recyclage en cours d'emploi}

Plus de la moitié des prestataires ont été recyclés en cours d'emploi sur la prévention des infections, le counselling/IEC IST/VIH/SIDA, la formation clinique en IST/VIH/SIDA, le partogramme, le PEV (vaccination des enfants et VAT) et la technique IEC (Tableau 15). Certains domaines tels que la surveillance nutritionnelle staturo-pondérale, la gestion de logistique contraceptive, la prévention et prise en charge de la malnutrition, la LMD, la technologie contraceptive, la SRAJ, la PCIME, l'allaitement maternel et la bonne pratique du sevrage, la CPN recentrée, la consultation de l'infertilité/infécondité et la consultation prénuptiale et préconceptionnelle n’ont pas fait l'objet de recyclage en cours d'emploi. Moins de 20 \% seulement des prestataires ont été recyclés sur ces thématiques.

Si la prévention des infections (PI) est abordée dans les formations de base en général, elle reste le principal thème de recyclage des prestataires en plus des IST/VIH/SIDA. Concernant les adolescents, il apparaît que moins de 3 prestataires sur 5 ont reçu une formation de base en SRAJ et moins de 1 prestataire sur 5 y a été recyclé. La question de la prise en compte des préoccupations des adolescents se pose assez clairement. 
Tableau 15 : Proportions par service des prestataires ayant reçu des compétences au cours de leur formation de base et de prestataires ayant reçu un recyclage en cours d'emploi par région

\begin{tabular}{|c|c|c|c|c|c|c|c|c|c|c|c|c|}
\hline \multirow[b]{2}{*}{ Domaines de formation } & \multicolumn{6}{|c|}{ Formation de base } & \multicolumn{6}{|c|}{ Recyclage } \\
\hline & $\begin{array}{l}\text { Centre- } \\
\text { nord }\end{array}$ & Sahel & Centre-est & $\begin{array}{l}\text { Centre- } \\
\text { sud }\end{array}$ & Est & Ensemble & $\begin{array}{l}\text { Centre- } \\
\text { nord }\end{array}$ & Sahel & $\begin{array}{l}\text { Centre- } \\
\text { est }\end{array}$ & $\begin{array}{l}\text { Centre- } \\
\text { sud }\end{array}$ & Est & Ensemble \\
\hline $\begin{array}{l}\text { Surveillance de Grossesse, Accouchement et soins du Post } \\
\text { Partum }\end{array}$ & 96,6 & 94,1 & 100,0 & 88,9 & 100,0 & 97,0 & 17,2 & 29,4 & 56,6 & 44,4 & 33,3 & 38,3 \\
\hline Prévention et Prise en charge de la malnutrition & 93,1 & 97,1 & 100,0 & 88,9 & 100,0 & 97,0 & 34,5 & 38,2 & 28,3 & 16,7 & 15,2 & 27,5 \\
\hline PEV (vaccination des enfants et VAT) & 93,1 & 97,1 & 98,1 & 88,9 & 97,0 & 95,8 & 58,6 & 52,9 & 47,2 & 61,1 & 45,5 & 51,5 \\
\hline Allaitement Maternel et Bonne Pratique du Sevrage & 93,1 & 94,1 & 100,0 & 88,9 & 97,0 & 95,8 & 17,2 & 11,8 & 20,8 & 22,2 & 9,1 & 16,2 \\
\hline Surveillance Nutritionnelle staturo-Pondérale & 96,6 & 91,2 & 100,0 & 88,9 & 93,9 & 95,2 & 51,7 & 35,3 & 26,4 & 22,2 & 15,2 & 29,9 \\
\hline LMD & 89,7 & 97,1 & 100,0 & 77,8 & 97,0 & 94,6 & 27,6 & 23,5 & 34,0 & 22,2 & 3,0 & 23,4 \\
\hline Technique IEC & 89,7 & 97,1 & 94,3 & 88,9 & 90,9 & 92,8 & 44,8 & 55,9 & 66,0 & 55,6 & 27,3 & 51,5 \\
\hline Prise en charge des cas d'avortement & 93,1 & 88,2 & 100,0 & 72,2 & 97,0 & 92,8 & 24,1 & 17,6 & 43,4 & 50,0 & 33,3 & 33,5 \\
\hline Prévention des infections & 96,6 & 97,1 & 96,2 & 72,2 & 87,9 & 92,2 & 72,4 & 73,5 & 71,7 & 88,9 & 66,7 & 73,1 \\
\hline Counselling/IEC IST/VIH/SIDA & 65,5 & 85,3 & 96,2 & 72,2 & 90,9 & 85,0 & 62,1 & 76,5 & 71,7 & 66,7 & 48,5 & 65,9 \\
\hline Formation clinique en IST/VIH/SIDA & 82,8 & 67,6 & 98,1 & 61,1 & 97,0 & 85,0 & 62,1 & 61,8 & 77,4 & 55,6 & 51,5 & 64,1 \\
\hline Soins Obstétricaux Essentiels (SOE) & 89,7 & 52,9 & 96,2 & 77,8 & 87,9 & 82,6 & 27,6 & 41,2 & 58,5 & 38,9 & 42,4 & 44,3 \\
\hline Consultation Infertilité/ infécondité & 65,5 & 64,7 & 73,6 & 72,2 & 72,7 & 70,1 & 3,4 & 5,9 & 3,8 & 0,0 & 9,1 & 4,8 \\
\hline SONU & 55,2 & 55,9 & 83,0 & 44,4 & 60,6 & 64,1 & 17,2 & 20,6 & 45,3 & 44,4 & 48,5 & 35,9 \\
\hline Partogramme & 55,2 & 52,9 & 56,6 & 33,3 & 84,8 & 58,7 & 34,5 & 52,9 & 56,6 & 55,6 & 60,6 & 52,7 \\
\hline Technologie contraceptive & 65,5 & 61,8 & 39,6 & 55,6 & 78,8 & 58,1 & 17,2 & 20,6 & 13,2 & 16,7 & 27,3 & 18,6 \\
\hline SRAJ & 41,4 & 58,8 & 58,5 & 66,7 & 60,6 & 56,9 & 17,2 & 5,9 & 32,1 & 22,2 & 6,1 & 18,0 \\
\hline Gestion de Logistique contraceptive & 44,8 & 35,3 & 43,4 & 44,4 & 63,6 & 46,1 & 20,7 & 17,6 & 37,7 & 38,9 & 24,2 & 28,1 \\
\hline Consultation prénuptiale et préconceptionnelle & 37,9 & 23,5 & 41,5 & 22,2 & 57,6 & 38,3 & 0,0 & 8,8 & 1,9 & 11,1 & 6,1 & 4,8 \\
\hline PCIME & 31,0 & 23,5 & 34,0 & 33,3 & 60,6 & 36,5 & 20,7 & 17,6 & 20,8 & 16,7 & 9,1 & 17,4 \\
\hline Technique de Counselling pour le test de dépistage & 27,6 & 44,1 & 13,2 & 33,3 & 48,5 & 31,1 & 58,6 & 52,9 & 54,7 & 55,6 & 18,2 & 47,9 \\
\hline CPN recentrée & 27,6 & 0,0 & 24,5 & 55,6 & 45,5 & 27,5 & 6,9 & 0,0 & 20,8 & 11,1 & 24,2 & 13,8 \\
\hline PTME & 3,4 & 17,6 & 15,1 & 27,8 & 48,5 & 21,6 & 34,5 & 38,2 & 30,2 & 44,4 & 30,3 & 34,1 \\
\hline Effectif de prestataires & 29 & 34 & 53 & 18 & 33 & 167 & 29 & 34 & 53 & 18 & 33 & 167 \\
\hline
\end{tabular}




\subsection{Pratiques en matière de $\mathbf{P F}$}

Des méthodes contraceptives sont habituellement prescrites aux clients par la majorité des prestataires (85,6 \%). Tous les prestataires l'ont fait dans la zone de l'Est. C'est au Centrenord que moins de prestataires le font (65,5\%). Les méthodes contraceptives les plus fournies par les prestataires au cours des six mois avant la collecte des données ont été les pilules (93,7 $\%)$ et les injectables (93,0 \%). Les autres méthodes sont moins fournies car moins de $50 \%$ des prestataires les ont offertes (Tableau 16). On remarque que la tendance des méthodes offertes est en sens inverse de la rupture de stock de ces mêmes méthodes. Il semblerait que les méthodes les moins offertes ont connu moins de ruptures de stocks.

Tableau 16 : Proportions des prestataires selon la méthode fournie les six derniers mois et proportions de clients observés en PF auxquels des méthodes de contraception ont été présentées par type de méthode

\begin{tabular}{|c|c|c|c|c|c|c|c|}
\hline \multirow[b]{2}{*}{ Méthodes de PF } & \multicolumn{6}{|c|}{ Fournies par le prestataire } & \multirow{2}{*}{$\begin{array}{c}\text { Présentées } \\
\text { aux clientes } \\
\text { de PF }\end{array}$} \\
\hline & $\begin{array}{c}\text { Centre- } \\
\text { nord }\end{array}$ & Sahel & $\begin{array}{c}\text { Centre- } \\
\text { est }\end{array}$ & $\begin{array}{l}\text { Centre- } \\
\text { sud }\end{array}$ & Est & Ensemble & \\
\hline Pilule Combinée & 100,0 & 100,0 & 84,8 & 100,0 & 93,3 & 93,7 & 51,7 \\
\hline Injectable & 100,0 & 86,7 & 93,5 & 100,0 & 90,0 & 93,0 & 51,7 \\
\hline Préservatifs Masculins & 52,6 & 53,3 & 46,7 & 33,3 & 56,7 & 49,3 & 37,9 \\
\hline MAMA & 36,8 & 10,0 & 44,4 & 72,2 & 63,3 & 43,7 & 13,8 \\
\hline Pilule Progestative & 78,9 & 36,7 & 28,3 & 16,7 & 56,7 & 41,3 & 41,4 \\
\hline Norplant & 15,8 & 33,3 & 55,6 & 11,1 & 36,7 & 35,9 & 51,7 \\
\hline Planification familiale naturelle & 15,8 & 13,3 & 37,8 & 38,9 & 50,0 & 32,4 & 17,2 \\
\hline Contraception d’urgence & 15,8 & 23,3 & 28,9 & 11,1 & 36,7 & 25,4 & - \\
\hline Préservatifs Féminins & 15,8 & 6,7 & 6,7 & 22,2 & 20,0 & 12,7 & - \\
\hline Spermicides & 10,5 & 6,7 & 6,7 & 0,0 & 30,0 & 11,3 & 27,6 \\
\hline DIU & 5,3 & 13,3 & 8,7 & 5,6 & 16,7 & 10,5 & 24,1 \\
\hline Méthode du collier ${ }^{3}$ & 0,0 & 3,3 & 6,8 & 0,0 & 6,7 & 4,3 & 10,3 \\
\hline Vasectomie & 0,0 & 3,3 & 2,3 & 0,0 & 0,0 & 1,4 & 17,2 \\
\hline Ligature des Trompes (LT) & 0,0 & 3,3 & 0,0 & 0,0 & 3,3 & 1,4 & - \\
\hline Effectif de prestataires & 29 & 34 & 53 & 18 & 33 & 167 & 76 \\
\hline
\end{tabular}

\section{Présentation des méthodes de planification familiale}

Pour permettre à la cliente de faire un choix éclairé, les méthodes contraceptives doivent être présentées aux clients tout en leur donnant des explications ou conseils. Au cours des observations de l'interaction client prestataire en consultation de PF, les méthodes ont été présentées par 51,7 \% des prestataires (Tableau 16). A l'exception de la pilule et de l'injectable, les autres méthodes sont moins présentées aux clientes par les prestataires. Pour certains prestataires (85,5\%), il y a des méthodes qu'ils ne recommandent pas aux adolescents. Au Centre-est tous les prestataires l'ont affirmé. Il s'agit surtout de la ligature des trompes, de la vasectomie, du DIU, de l'injectable et du norplant (Graphique 10).

\footnotetext{
${ }^{3}$ Méthode du collier était en phase d'extension
} 
Graphique 10 Proportions des prestataires par méthode contraceptive qu'ils ne recommanderaient pas pour les adolescents

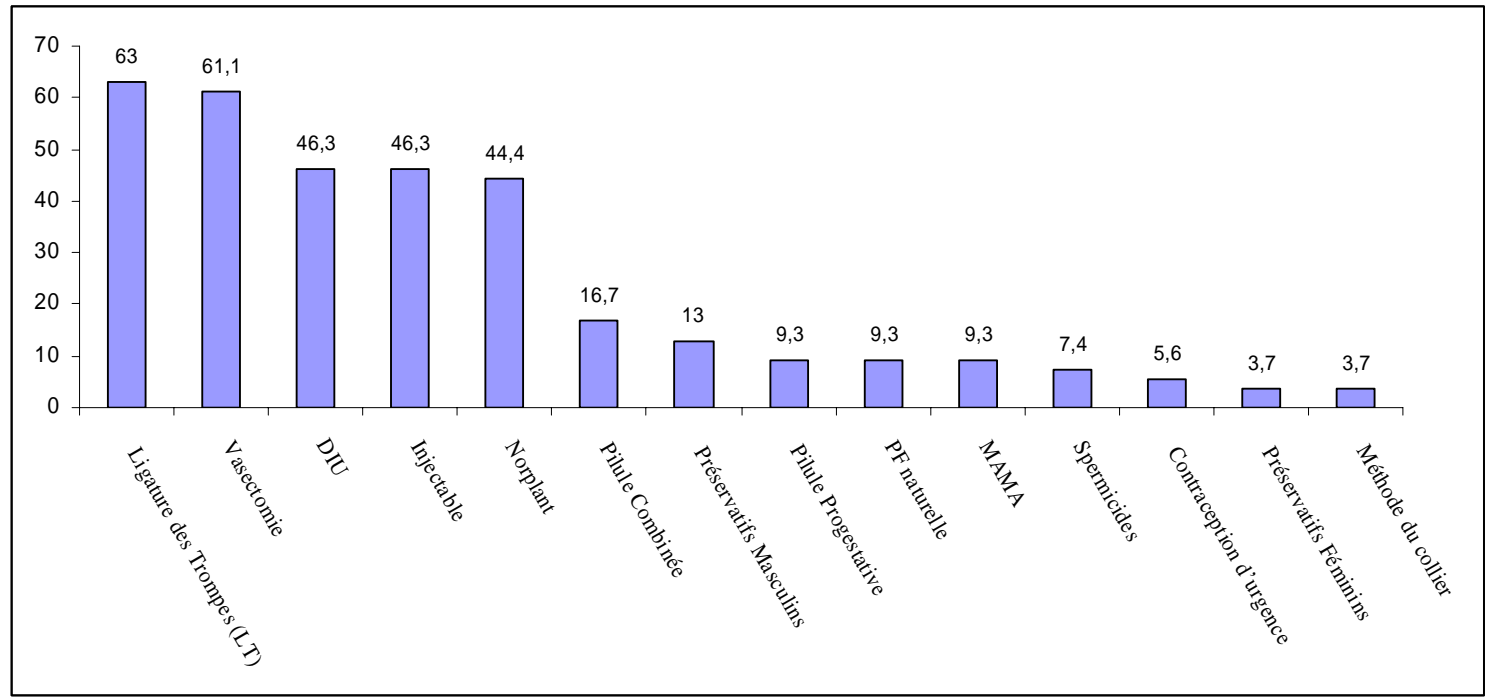

\section{Relations interpersonnelles et fourniture de méthodes contraceptives}

Certains critères, barrières, restrictions ou exigences érigés par les prestataires limitent l'accès des clientes aux méthodes contraceptives. Non contenus dans les normes et protocoles, ils n’ont pas de fondement médical. Nous nous intéresserons à ceux liés à l'âge et à la situation matrimoniale.

\section{Selon un âge minimum}

Les prestataires interviewés ont affirmé ne pas offrir toute la gamme des méthodes contraceptives aux clients en dessous d'un âge minimum. Plus de la moitié des prestataires ne fournissent pas la pilule et les injectables aux clients en dessous d'un âge minimum (tableau 17). Cet âge minimum moyen est de 10 ans pour presque toutes les méthodes. Pour plus du quart des prestataires l'offre de préservatif (masculin et féminin) connaît aussi des restrictions. Cela est très inquiétant dans un contexte de lutte contre le VIH/SIDA. Il en est de même des spermicides, de la PF naturelle, de la contraception d'urgence et de la méthode du collier (Tableau 17).

Tableau 17 : Proportions des prestataires observant certaines restrictions par méthode contraceptive

\begin{tabular}{|c|c|c|c|c|c|c|}
\hline \multirow{2}{*}{ Méthodes } & \multicolumn{4}{|c|}{ Age } & \multirow{2}{*}{$\begin{array}{c}\text { Selon le statut matrimonial } \\
\text { de célibataire }\end{array}$} & \multirow{2}{*}{$\begin{array}{c}\text { Exigeant l'approbation } \\
\text { du mari avant l'offre }\end{array}$} \\
\hline & $\%$ & Min & $\%$ & Max & & \\
\hline Pilule Combinée & 59,9 & 10 & 46,5 & 60 & 84,5 & 16,9 \\
\hline Pilule Progestative & 54,2 & 10 & 43,7 & 60 & 74,6 & 14,8 \\
\hline DIU & 37,3 & 10 & 35,9 & 60 & 63,4 & 17,0 \\
\hline Injectable & 58,5 & 10 & 45,8 & 60 & 71,8 & 16,9 \\
\hline Norplant & 40,8 & 10 & 36,6 & 60 & 71,8 & 19,7 \\
\hline Préservatifs Féminins & 28,9 & 10 & 17,6 & 60 & 81,7 & 17,6 \\
\hline Préservatifs Masculins & 29,6 & 10 & 14,8 & 90 & 83,8 & 18,3 \\
\hline Spermicides & 25,4 & 10 & 23,2 & 60 & 78,9 & 21,1 \\
\hline Ligature des Trompes (LT) & 29,6 & 12 & 26,8 & 60 & 22,5 & 39,7 \\
\hline Vasectomie & 27,5 & 12 & 23,9 & 70 & 23,2 & 36,2 \\
\hline Planification Familiale naturelle & 33,1 & 10 & 27,5 & 60 & 74,6 & 21,1 \\
\hline MAMA & 27,5 & 10 & 28,2 & 55 & 69,7 & 13,4 \\
\hline Contraception d'urgence & 24,6 & 10 & 23,9 & 55 & 72,5 & 16,2 \\
\hline Méthode du collier & 15,5 & 10 & 22,5 & 55 & 63,4 & 14,2 \\
\hline
\end{tabular}




\section{Selon un âge maximum}

En général, moins de 2 prestataires sur 5 ne fournissent pas de méthodes contraceptives aux clients au-dessus d'un âge maximum de 60 ans en moyenne. Cet âge est moins variable selon la méthode.

\section{Selon le statut matrimonial}

Pour plus de 3 prestataires sur 4, le célibat constitue une barrière à la prescription des méthodes contraceptives (Tableau 17). C’est seulement pour les méthodes définitives qu'un peu plus de 1 prestataire sur 5 considèrent le célibat comme une barrière à la prescription. Très peu de prestataires exigent l'approbation du mari avant de fournir à une femme qui le désire une méthode contraceptive. C'est encore pour les méthodes définitives que l'on observe que plus de prestataires exigent l'approbation du mari.

\subsection{Connaissances des services et fréquentation des FS}

La connaissance des services disponibles dans les FS est une des conditions de l'utilisation des services de santé par les clients. Pour plus de la moitié d'entre eux, la consultation prénatale, l'accouchement, les services curatifs - adulte et les services curatifs - enfant sont les services connus par les clients (Tableau 18).

Tableau 18 : Proportion des clients selon la connaissance des services offerts dans les FS par région

\begin{tabular}{lcccccc}
\hline Services offerts connus & $\begin{array}{c}\text { Centre- } \\
\text { nord }\end{array}$ & Sahel & $\begin{array}{c}\text { Centre- } \\
\text { est }\end{array}$ & $\begin{array}{c}\text { Centre- } \\
\text { sud }\end{array}$ & Est & Ensemble \\
\hline Consultation prénatale & 74,1 & 85,7 & 91,8 & 92,7 & 66,7 & 82,7 \\
Accouchement & 70,4 & 69,0 & 83,6 & 68,3 & 63,3 & 72,0 \\
Services curatifs-adulte & 85,2 & 50,0 & 76,7 & 85,4 & 55,0 & 69,1 \\
Services curatifs-enfant & 77,8 & 40,5 & 82,2 & 56,1 & 70,0 & 67,1 \\
Planification Familiale & 44,4 & 42,9 & 61,6 & 26,8 & 31,7 & 43,2 \\
Vaccination de l'enfant & 40,7 & 28,6 & 63,0 & 39,0 & 31,7 & 42,8 \\
Consultation Postnatale & 44,4 & 19,0 & 60,3 & 19,5 & 13,3 & 32,9 \\
LMD (Réhydratation par voie orale) & 37,0 & 7,1 & 65,8 & 7,3 & 20,0 & 31,3 \\
Traitement MST autres que VIH/SIDA & 7,4 & 2,4 & 34,2 & 0,0 & 5,0 & 12,8 \\
Surveillance nutritionnelle et pondérale & 3,7 & 2,4 & 24,7 & 4,9 & 8,3 & 11,1 \\
Conseil en Nutrition & 7,4 & 2,4 & 23,3 & 4,9 & 1,7 & 9,5 \\
Diagnostic IST autres que VIH/SIDA & 3,7 & 0,0 & 21,9 & 0,0 & 1,7 & 7,4 \\
Counselling/IEC VIH/SIDA & 11,1 & 2,4 & 6,8 & 9,8 & 5,0 & 6,6 \\
Counselling/IEC IST autres que VIH/SIDA & 3,7 & 2,4 & 11,0 & 2,4 & 0,0 & 4,5 \\
Soins post-abortum & 14,8 & 0,0 & 9,6 & 0,0 & 0,0 & 4,5 \\
PTME & 11,1 & 0,0 & 6,8 & 2,4 & 0,0 & 3,7 \\
TEST VIH/SIDA & 7,4 & 0,0 & 4,1 & 2,4 & 0,0 & 2,5 \\
Consultation infécondité/infertilité & 7,4 & 0,0 & 2,7 & 0,0 & 0,0 & 1,6 \\
Prise en charge SR des adolescents & 7,4 & 0,0 & 1,4 & 0,0 & 1,7 & 1,6 \\
\hline Effectif de clients & $\mathbf{2 7}$ & $\mathbf{4 2}$ & $\mathbf{7 3}$ & $\mathbf{4 1}$ & $\mathbf{6 0}$ & $\mathbf{2 4 3}$ \\
\hline
\end{tabular}

Les autres services tels que la prise en charge SR des adolescents, la consultation infécondité/infertilité, le test du VIH/SIDA, la PTME, les soins post-abortum, le counselling/IEC/IST autres que VIH/SIDA, le counselling/IEC/VIH/SIDA, le diagnostic des IST autres que les IST/VIH/SIDA et le conseil en nutrition ne sont pratiquement pas connus 
(moins de $10 \%$ ). La prise en charge de la SR des adolescents est un service totalement ignoré $(1,6 \%)$ pour l'ensemble des clients.

Au cours des 12 derniers mois précédant l'enquête, environ 3 femmes sur 4 et 3 hommes sur 5 ont été en consultation. Cette fréquentation est variable d'une région à l'autre (Graphique 11. A l'Est surtout, la fréquentation est la plus faible. Les hommes fréquentent moins les FS comparativement aux femmes.

\section{Graphique 11: Proportions de femmes et d'hommes ayant été en consultation par région}

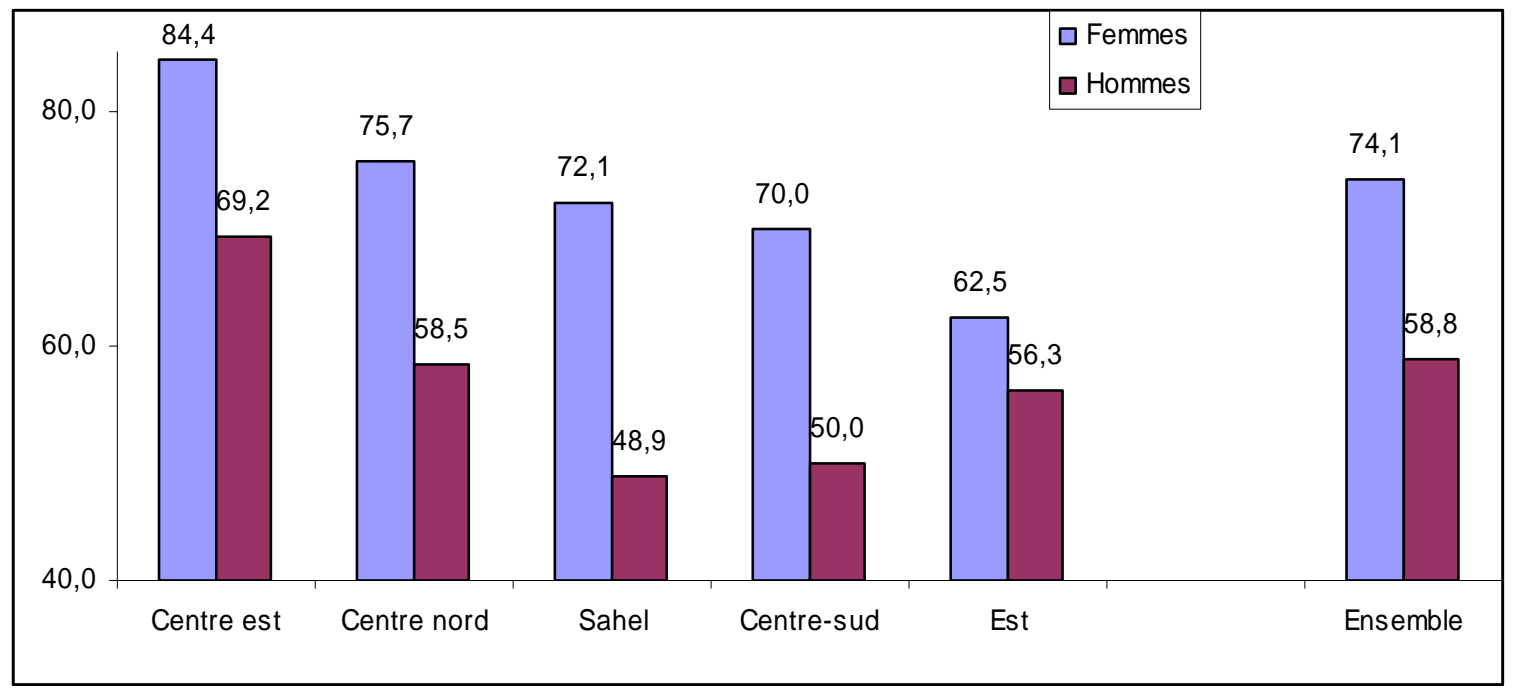

Au cours des 12 derniers mois précédents l'enquête, ce sont les soins aux enfants qui ont amené les femmes en consultation. Il s'agit du traitement des maladies infantiles et de la vaccination d'enfants où 2/3 des femmes sont concernées (Graphique 12. Il y a eu 1/3 des femmes qui ont consulté pour les soins prénataux. Les accouchements et les soins post-natals (45 jours après l'accouchement) ont concerné environ une femme sur 4. 
Graphique 12: Proportions des femmes selon l'utilisation des services au cours des 12 derniers mois

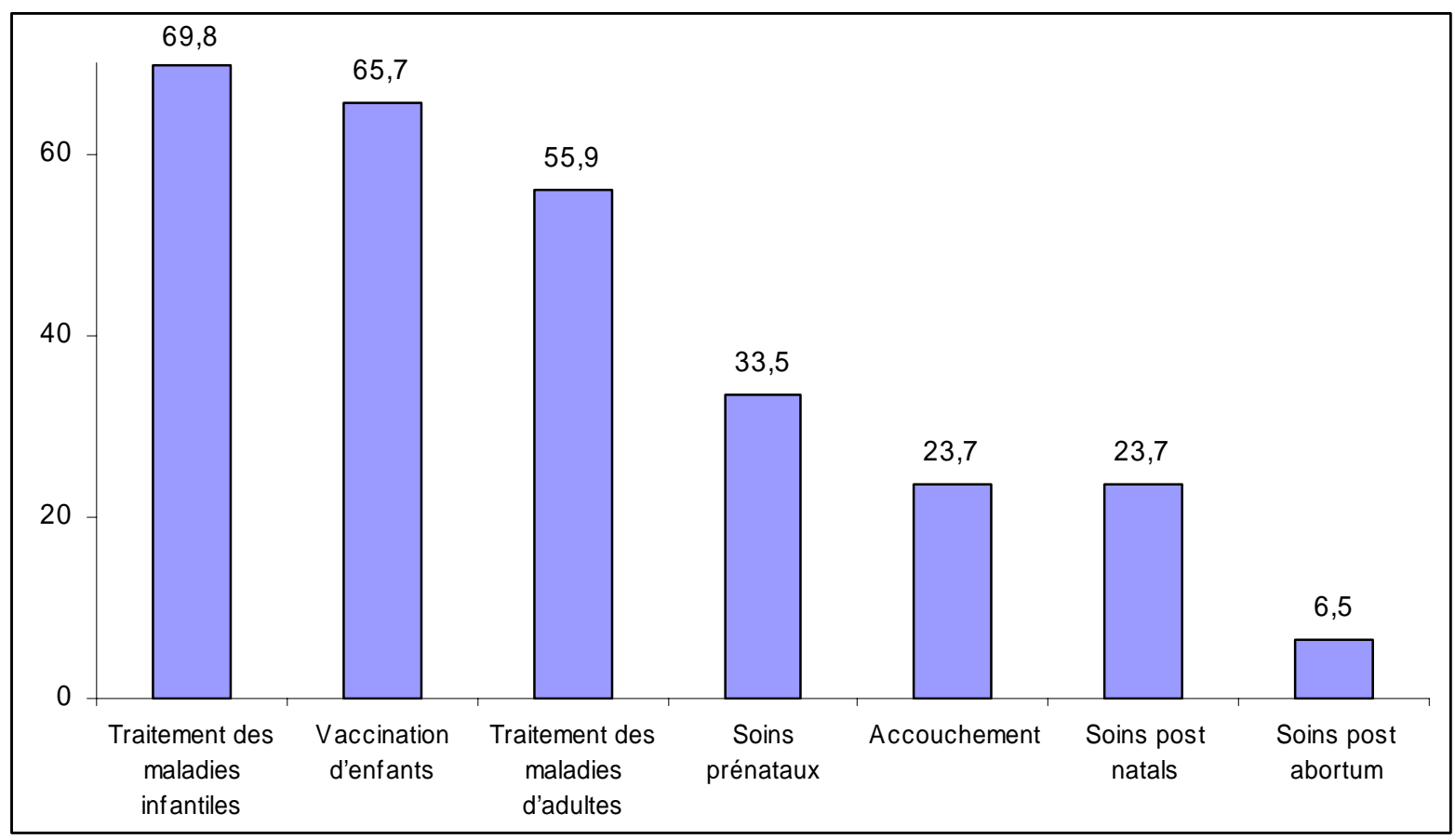

Les adolescentes ont été proportionnellement plus nombreuses que les adultes et les adolescents à avoir fréquenté une FS au cours des 12 derniers mois précédents la collecte des données (Graphique 13. Un tiers seulement des adolescents a fréquenté une FS au cours des 12 derniers mois précédents la collecte des données.

\section{Graphique 13: Proportions de femmes et d'hommes ayant été en consultation}

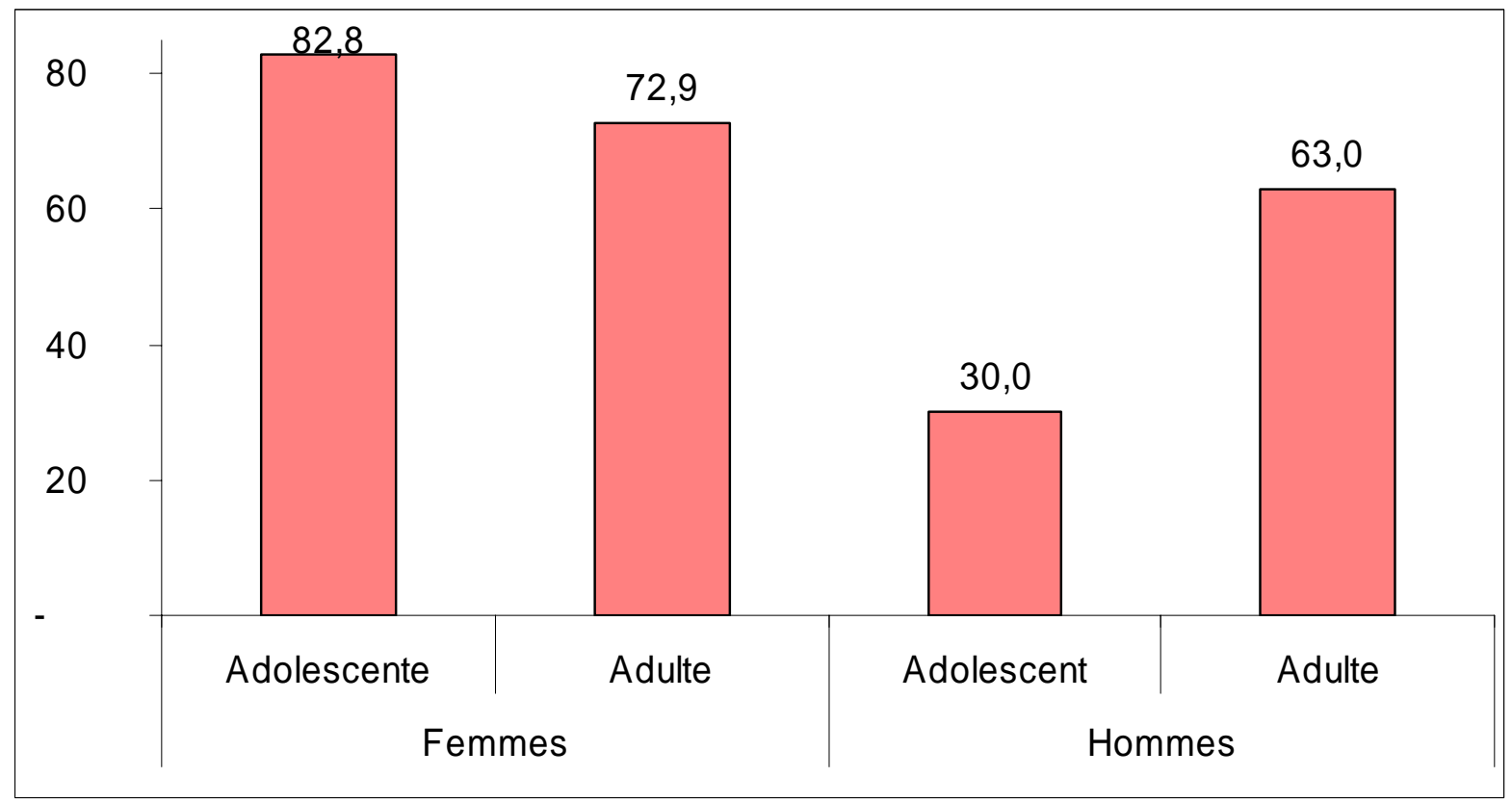




\section{Utilisation des services de ASC/AV}

Plus de la moitié des femmes $(57,7 \%)$ et des hommes $(64,1 \%)$ adultes reconnaissent l'existence d'un agent de santé communautaire (ASC) dans leur localité. Les adolescentes particulièrement le reconnaissent moins. S'agissant du recours à l'ASC, il faut noter cependant que les femmes particulièrement, et surtout les adolescentes y ont moins recours (Graphique 14. En effet, c'est un quart des adolescentes parmi la moitié qui connaissent sa présence qui se réfère à l'ASC. Toutes les femmes et tous les hommes ont eu recours à un professionnel de santé plutôt qu'à l'accoucheuse villageoise (AV) au cours des 12 derniers mois avant l'enquête. L'AV n'est pratiquement pas sollicitée par eux.

Graphique 14: Proportions de femmes et d'hommes connaissant et utilisant les services $\underline{\text { d'ASC }}$

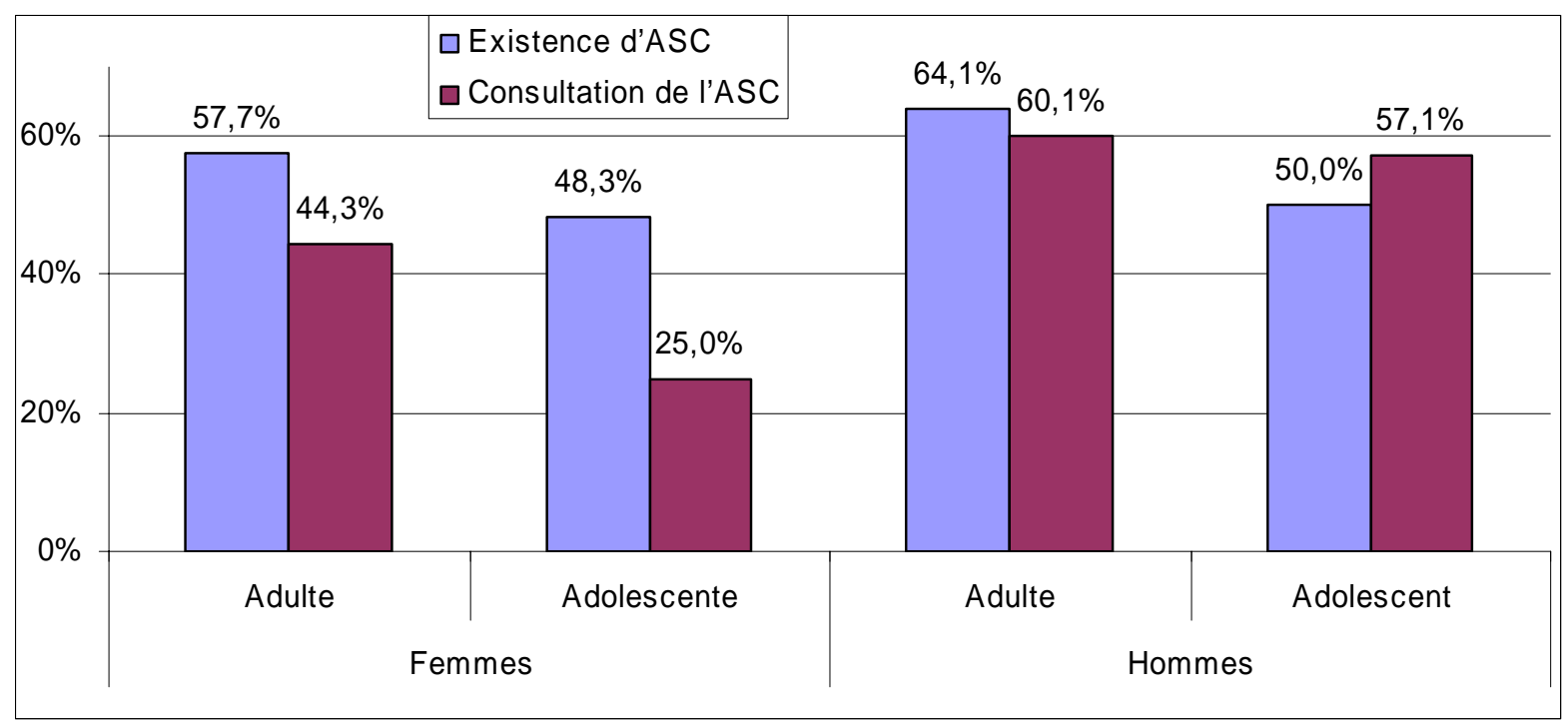

\section{Raisons de la fréquentation ou non des services}

La fréquentation des FS est conditionnée par plusieurs facteurs. Il ressort de la présente analyse que c'est essentiellement les coûts de prestations qui justifient la non fréquentation des services. Plus de 50 \% des femmes, de même que des hommes l'ont affirmé. L'éloignement de la FS, le mauvais accueil, l'insatisfaction des services et le recours au tradipraticien ne sont pas des raisons majeures de non fréquentation des services car moins de $10 \%$ des femmes et des hommes l’ont affirmé.

Pour 78,6 \% des femmes et 66,7 \% des adolescentes, elles ont été satisfaites de l'accueil quand elles ont eu recours aux services pour un accouchement. Ils sont 86,1\% des hommes à exprimer également leur satisfaction.

Selon le tiers des femmes et des hommes, aussi bien adultes qu'adolescents, l'accueil et la conviction de l'efficacité des services à résoudre les problèmes sont les principales motivations pour le choix d'une FS lorsqu'il s'agit d'obtenir une méthode de PF. 


\subsection{Connaissances de la communauté sur la SR}

Il s’agit ici de la connaissance de la communauté sur certains thèmes de la SR, plus spécifiquement des risques liés à la grossesse et à l'accouchement.

\section{Connaissances des facteurs de risques de la grossesse}

Pour plus de la moitié des hommes et des femmes, le jeune âge de la femme enceinte est un facteur de risque (Tableau 19). A cela, il faudra ajouter les grossesses rapprochées et les grossesses multiples selon les femmes. Les autres facteurs sont cités dans une moindre mesure.

\section{Signes de complication d'une grossesse}

Les signes de complication d'une grossesse sont très peu connus aussi bien par les femmes que les hommes. On note qu'en général, moins de 1 personne sur 4 connaît ces signes. Les vertiges, la forte fièvre, l'anémie ou la pâleur et les membres ou visage enflés sont prépondérants dans les réponses. Les saignements vaginaux au cours de la grossesse et l’immobilité du fœtus en particulier semblent ne pas constituer un signe d'alerte.

Tableau 19 : Proportions des femmes et proportions des hommes selon les facteurs de risque de la grossesse et selon les signes de complications du travail et d'une grossesse

\begin{tabular}{|c|c|c|}
\hline Facteurs de risque pour une femme si elle tombe enceinte revoir titre & Femmes & Hommes \\
\hline Jeune âge & 63,9 & 61,7 \\
\hline Grossesses rapprochées & 59,9 & 49,5 \\
\hline Grossesses multiples & 50,3 & 49,5 \\
\hline Petite taille & 38,1 & 35,5 \\
\hline Age avancé & 34,7 & 30,8 \\
\hline Ancienne opérée & 32,0 & 34,6 \\
\hline Boiterie & 29,3 & 29,9 \\
\hline Avortements antérieurs & 25,9 & 27,1 \\
\hline Autres & 14,3 & 19,8 \\
\hline Mort-né antérieur & 10,2 & 23,4 \\
\hline \multicolumn{3}{|l|}{ Symptômes ou signes de complications d'une grossesse } \\
\hline Vertiges & 27,2 & 21,1 \\
\hline Forte fièvre & 26,7 & 18,1 \\
\hline Anémie/pâleur & 24,3 & 22,1 \\
\hline Jambes, bras ou visages enflés & 20,6 & 24,0 \\
\hline Saignements vaginaux au cours de la grossesse & 18,1 & 16,7 \\
\hline Maux de tête/Céphalées & 14,4 & 11,3 \\
\hline Fœtus ne bouge pas & 5,3 & 5,4 \\
\hline Urines troubles & 0,8 & 1,5 \\
\hline Autres & 32,5 & 30,9 \\
\hline NSP & 17,3 & 27,9 \\
\hline \multicolumn{3}{|l|}{ Signes de reconnaissance du travail chez la femme enceinte } \\
\hline Douleurs des reins/bas ventre & 69,5 & 61,0 \\
\hline Contractions utérines & 14,4 & 9,5 \\
\hline Autres & 8,2 & 7,5 \\
\hline NSP/NR & 7,8 & 22,0 \\
\hline Total & 100,0 & 100,0 \\
\hline \multicolumn{3}{|l|}{ Signes de danger du travail/Accouchement } \\
\hline Durée prolongée du travail & 48,3 & 56,9 \\
\hline Hémorragie pendant le travail & 27,5 & 24,6 \\
\hline Saignement après accouchement & 14,2 & 6,2 \\
\hline
\end{tabular}




\begin{tabular}{lcc} 
Présentation non céphalique & 6,7 & 3,1 \\
Autres & 3,3 & 9,2 \\
\hline Total & 100,0 & 100,0 \\
\hline Effectif & 245 & 238 \\
\hline
\end{tabular}

\section{Signes de complication du travail lors de l'accouchement}

Les douleurs des reins et/ou du bas-ventre sont les signes connus des femmes et des hommes comme éléments annonciateurs du travail (Tableau 19). La durée prolongée du travail est un signe important de danger pendant le travail de la femme enceinte. Le saignement après l'accouchement et la présentation non céphalique ne sont pas considérés comme très préoccupants car moins de femmes et d’hommes y ont fait référence. 


\section{CONCLUSION}

L'analyse secondaire à partir de la base de données de l'analyse situationnelle des services de SR de 2006 fait un état des lieux de la fonctionnalité et de la qualité des services de santé offerts par les FS de la zone d'intervention du projet «Eliminer le mariage précoce au Burkina».

S’agissant de la capacité fonctionnelle des services de SR, leur effectivité est affirmée par la disponibilité des services. La PCIME, la récupération nutritionnelle, les tests VIH/SIDA, la PTME, le diagnostic au laboratoire des IST et la prise en charge pédiatrique du VIH sont des services moins présents dans les FS.

Les infrastructures ne sont pas disponibles dans toutes les FS. Les toilettes ou latrines, les sources d'eau, les systèmes de traitement des déchets et les dispositifs d'évacuation des eaux usées sont absents dans nombre de FS. Les caractéristiques des salles d'examen ne répondent pas quelquefois aux normes en terme de discrétion et d'éclairage. Les insuffisances liées aux infrastructures sont aussi observées dans les équipements et le matériel. En effet, les kits d'accouchement tout comme les ballons et masque pour la réanimation néonatale manquent dans les FS.

Au total, les normes de fonctionnement ne sont pas remplies en termes d'infrastructures, d'équipement, de matériel et de ressources humaines. De nombreux CSPS ne disposent pas du personnel requis pour offrir les différents services. La gestion des services montrent aussi des insuffisances par les ruptures de stocks de médicaments et consommables observées, et par les insuffisances de la supervision.

Les services disponibles dans les FS ne sont pas bien connus par les populations. Ce qui peut limiter la fréquentation. Il apparaît aussi que les services offerts aux adolescents ne sont pas assez développés car peu de prestataires ont des compétences et de nombreuses FS n’en disposent pas. 



\section{RECOMMANDATIONS}

L'analyse secondaire a souligné des domaines ou aspects de la Santé de la Reproduction qui nécessitent des améliorations. Elles sont relatives à la capacité d'offre de services et à la qualité des services. Des recommandations sont formulées pour répondre aux problèmes ou obstacles identifiés au cours de l'analyse. Le projet doit tenir compte de la situation actuelle de chaque formation sanitaire avant toute action.

1. Il est ressorti que près de la moitié des CSPS de la zone d’intervention ne répondent pas aux normes en personnel en qualité et en quantité. Il serait donc urgent de les normaliser par une affectation de ressources humaines requises pour leur bon fonctionnement et par conséquent pour améliorer les offres de services. De plus, un redéploiement du personnel de santé pour palier le manque ou l'insuffisance du personnel de santé dans certaines FS serait une alternative.

2. Face aux ruptures de stock enregistrées, il est urgent que les services soient mieux organisés et mieux gérés pour garantir en permanence l'offre de produits et de services SR. La qualité des ressources humaines et les supervisions ordinaires contribueraient à réduire ces insuffisances. La validation du plan de sécurisation des produits de la SR entreprise par la DSF est une solution palliative aux ruptures de stocks lorsqu'elle sera mise en œuvre. Il faudra donc travailler à rendre ce plan effectif dans la zone d'intervention.

3. Les infrastructures et les équipements n'étant pas totalement disponibles dans les différentes FS, il y a lieu de les doter du minimum nécessaire pour leur meilleur fonctionnement. On citera entre autre la nécessité d'améliorer certaines commodités telles que les toilettes ou latrines, les systèmes de traitement des déchets et des eaux usées, la normalisation des caractéristiques des salles d'examen en terme d'éclairage et de discrétion.

4. L'absence de COGES fonctionnel dans certaines FS demande à être corrigée par leur mise en place dans les FS qui n'en disposent pas. Cela contribuera à une meilleure gestion dans les FS.

5. Face aux insuffisances constatées lors des interrogatoires et des examens physiques, de même que celles de la supervision, il serait urgent de prendre des dispositions pour rendre effectives les supervisions.

6. Dans l'organisation des services et la prise en charge des clients, par rapport à la non disponibilité et la non utilisation des documents de référence en SR constatée, il faudra:

a. Rendre disponibles les documents de référence de SR pour la planification et l'organisation des services (PS/MMR, PS/santé des jeunes, PCIME, PTME)

b. Doter toutes les structures en directives et protocoles pour permettre aux prestataires d'améliorer la qualité de la prise en charge.

7. Pour la gestion et les stratégies de collecte des données, il faudra veiller à ce que les registres soient bien tenus pour un meilleur suivi des FS 
8. L'harmonisation des coûts de service sera une réponse à la variabilité des coûts d'une FS à l'autre. Il est inadmissible que pour un même service, il y ait des différences d'une FS à l'autre. Ces différences peuvent être un facteur de fréquentation différentielle ou préférentielle des formations sanitaires.

9. Pour les services aux adolescents, il y a lieu non seulement d'élargir leur gamme, mais surtout de disposer des ressources humaines à même d'offrir ces services. De plus les préoccupations des adolescents sont à considérer pour leur offrir des services adaptés.

10. S'agissant des services nouveaux ou en phase d'extension, la nécessité de mise à niveau des prestataires s’impose.

11. La non disponibilité et le non usage du matériel IEC pose un problème d'éthique en terme de libre choix. Une insistance auprès des prestataires pour rendre disponible et pour une utilisation du matériel IEC est impérative. 


\section{BIBLIOGRAPHIE}

1 Analyse Situationnelle des services de santé de la reproduction au Burkina Faso, Décembre 2006, Population Council/DSF

2 Analyse Situationnelle des services de SR dans la zone d'intervention du FNUAP, Population Council, novembre 2000 -. - Mar Mamadou.- Sankara Olga - Kaboré Idrissa

3 Analyse Situationnelle des services de SR dans la zone d'intervention du projet UNF, Population Council, février 2002 -. - Dr Cissé Mahamadi, Lydia Saloucou - Mar Mamadou.

4 Enquête Démographique et de Santé 2003 INSD - Burkina Faso

5 Manuel de Recherche opérationnelle en matière de planification familiale - seconde édition - Andew Fisher, John E. Laing, John E. Stoeckel et John W. Townsend

6 Statistiques Sanitaires 2004 DEP/Santé 